\title{
Reflectance Spectral Features and Significant Minerals in Kaishantun Ophiolite Suite, Jilin Province, NE China
}

\author{
Chenglong Shi ${ }^{1}{ }^{(\mathbb{D}}$, Xiaozhong Ding $^{1, *}$, Yanxue Liu ${ }^{1}$ and Xiaodong Zhou ${ }^{2}$ \\ 1 Institute of Geology, Chinese Academy of Geological Sciences, No. 26 Baiwanzhuang Street, \\ Beijing 100037, China; sc10322@gmail.com (C.S.); lyxue@sohu.com (Y.L.) \\ 2 Survey of Regional Geological and Mineral Resource of Jilin Province, No.4177 Chaoda Road, \\ Changchun 130022, China; xiaodongzhou63@sohu.com \\ * Correspondence: dingxiaozhong@cags.ac.cn; Tel.: +86-10-6899-9675
}

Received: 28 January 2018; Accepted: 28 February 2018; Published: 5 March 2018

\begin{abstract}
This study used spectrometry to determine the spectral absorption of five types of mafic-ultramafic rocks from the Kaishantun ophiolite suite in Northeast China. Absorption peak wavelengths were determined for peridotite, diabase, basalt, pyroxenite, and gabbro. Glaucophane, actinolite, zoisite, and epidote absorption peaks were also measured, and these were used to distinguish such minerals from other associated minerals in ophiolite suite samples. Combined with their chemical compositions, the blueschist facies (glaucophane + epidote + chlorite) and greenschist facies (actinolite + epidote + chlorite) mineral assemblage was distinct based on its spectral signature. Based on the regional tectonic setting, the Kaishantun ophiolite suite probably experienced the blueschist facies metamorphic peak during subduction and greenschist facies retrograde metamorphism during later slab rollback.
\end{abstract}

Keywords: reflectance spectrum; ophiolite suite; mineral classification; Kaishantun; NE China

\section{Introduction}

Ophiolites are segments of oceanic crust that have been residually accreted in convergent boundaries [1-3]. The principal focus of recent studies related to ophiolites has been on the spatial and temporal patterns of felsic to mafic-ultramafic rock suites. These patterns are generated through processes of magmatic differentiation and separate melting episodes in specific tectonic settings [1-4]. The basic assumption is that the type and characteristics of a given ophiolite will be linked to its geological environment.

Mineral assemblages that include olivine, pyroxene, hornblende, etc. are helpful for ophiolite type discrimination. Unfortunately, due to severe alteration, the mineralogical compositions of many ophiolite samples are difficult to recognize in the field. The traditional techniques that have been used to resolve this problem, such as microscopic identification analysis and dissolution methods, typically require samples to be removed from an outcrop and separated into its constituent minerals, which makes the process inefficient and difficult. This limits the ability to perform ophiolite mineral component discrimination.

Multispectral remote sensing has already been effectively utilized to identify different lithologies and altered minerals [4-7]. This technique may be a feasible substitution for the recognition of olivine and its associated minerals, such as pyroxene, chlorite, and epidote, in their solid state. Electromagnetic radiation emitted by the sun interacts with materials and can be detected by remote sensors, which can determine its spectral pattern. This "material fingerprint" can be used to distinguish similar minerals and to determine their compositions [7]. 
In this paper, we assess the possibility of rock-mineral classification and ophiolite types identification by using hyperspectral remote-sensing data and discuss its geological significance. To this end, we first report the spectral characteristics of different parts of rock samples from the ophiolite suite in the Kaishantun area, Jilin Province, China. We then combine these results with mineral assemblage features and chemical compositions to test the ability of hyperspectral remote sensing to class and identify minerals in ophiolites.

\section{Geological Setting}

Northeast China, which has traditionally been regarded as the eastern part of the Central Asian Orogenic Belt (CAOB), is located between the North China Craton and Siberian Craton. Previous studies have shown two major stages of orogenic evolution in this complicated tectonic unit $[8,9]$ : the closure of the Paleo-Asian Ocean and the subsequent subduction of the Paleo-Pacific plate.

Numerous terranes and micro-continent fragments were grafted together in Northeast China during the Paleozoic, as the Paleo-Asian Ocean closed [10]: Sikhote-Alin Accretion and Bureya, Jiamusi, and Khaka Terranes (SA, BT, JT and KT) in the southeast; the Songliao and Xing'an blocks (SL and $\mathrm{XA}$ ) in the central area; and the Erguna block (EG) in the northwest (Figure 1). The EG is assumed to be the eastern part of the Central Mongolian micro-continent fragment, but its geologic and tectonic evolution has remained unclear due to extensive forest cover in the area [11,12]. Although accurate geologic age data have not been reported in previous studies, it is generally accepted that strata and intrusive rocks from the Proterozoic to Paleozoic are widespread in this area [13]. Dating of the EG with $\mathrm{Nd}$ isotope measurements of its granite suggests that the crust formed from 1680 to $1060 \mathrm{Ma}$, making it older than the SL or XA [14,15].

The formation of the Sikhote-Alin Accretion and the Bureya, Jiamusi, and Khaka Terranes in Northeast China resulted from the westward subduction of the Paleo-Pacific after the Mesozoic [16]. The Heilongjiang Complex and the Mashan Complex are two of three main components of the Jiamusi Terrane, with the other being an assortment of Permian granitoids. The Heilongjiang Complex is made up of mylonitic schists, carbonates, blueschist-facies metamorphosed rock, and mafic-ultramafic rocks. This complex, which has been identified as a mélange along the suture zone between the SL and Jiamusi Terrane, is distributed in the western part of the terrane [17-20]. The main petrofacies in the Jiamusi Terrane are deformation granites associated with the Mashan Complex (500-Ma-old) [9,17].

The Kaishantun area, which belongs to Yanji City, China, is not far from the border between North Korea, Russia, and China. The Sea of Japan is $\approx 100 \mathrm{~km}$ to the east of this area. The closure of the Paleo-Asian Ocean and the subsequent continent-continent collision led to this area becoming a part of CAOB between the North China Craton and the Siberian Craton (Figure 2b) $[8,9,13,17]$. The basement of the Kaishantun area can be divided into highly metamorphosed greenschist and amphibolite facies of Archean-to-Proterozoic granitic rocks [13]. The Paleozoic sedimentary strata, which are dominated by a distribution of converted shallow marine-to-paralic facies, were deposited near the end of Permian [13]. These strata were extensively intruded by a series of mafic-ultramafic rocks, with the largest being the Kaishantun ophiolite suite. Previous research has indicated that the formation of ophiolite suite connected with the closure of the Paleo-Asian Ocean and NE China continental accretion [21,22]. 


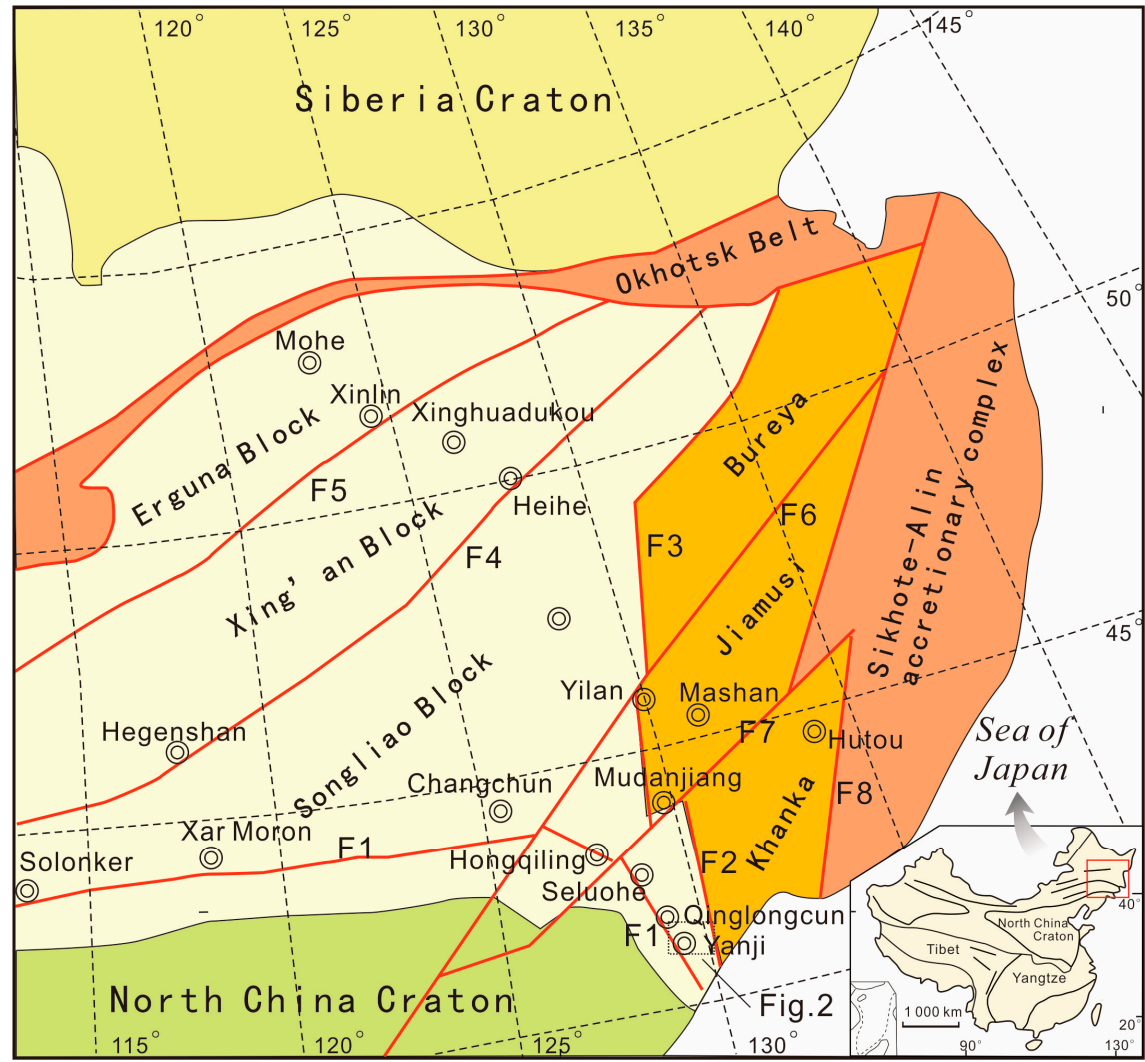

Figure 1. Tectonic sub-divisions of Northeast China and Far East Russia (after $[8,9]) . F 1=$ Xar Moron-Changchun-Yanji Fault; F2 = Mudanjiang Fault; F3 = Heilongjiang Fault; F4 = Hegenshan-Heihe Fault; F5 = Xinlin-Xiguitu Fault; F6 = Yilan-Yitong Fault; F7 = Dunhua-MishanFault; and F8 = Primoria Fault.
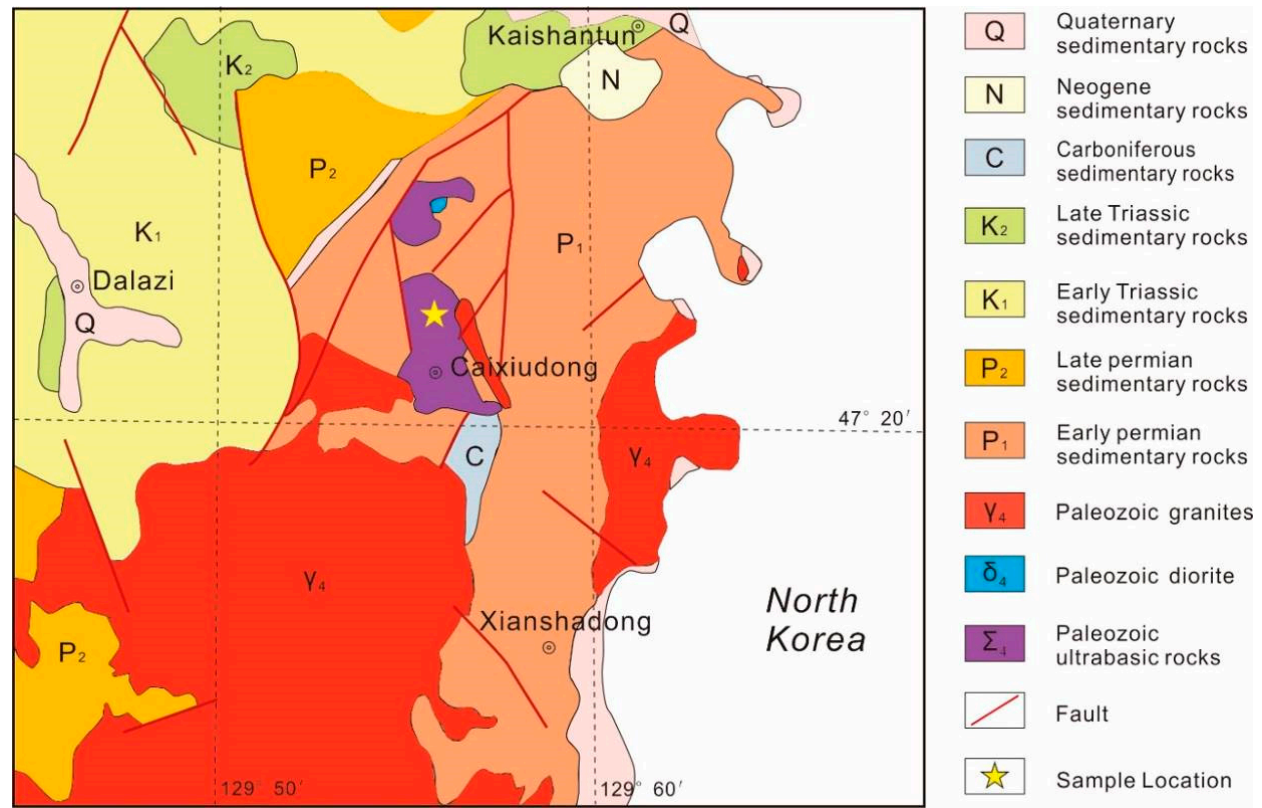

Figure 2. Simplified geological maps of the Kaishantun area (after [8,9]). 


\section{Samples and Methods}

We collected rock samples from the Caixiudong part of the Kaishantun area for our analysis. Photographs and sample locations of representative specimens are displayed in Figure 3 and Table 1 , respectively.
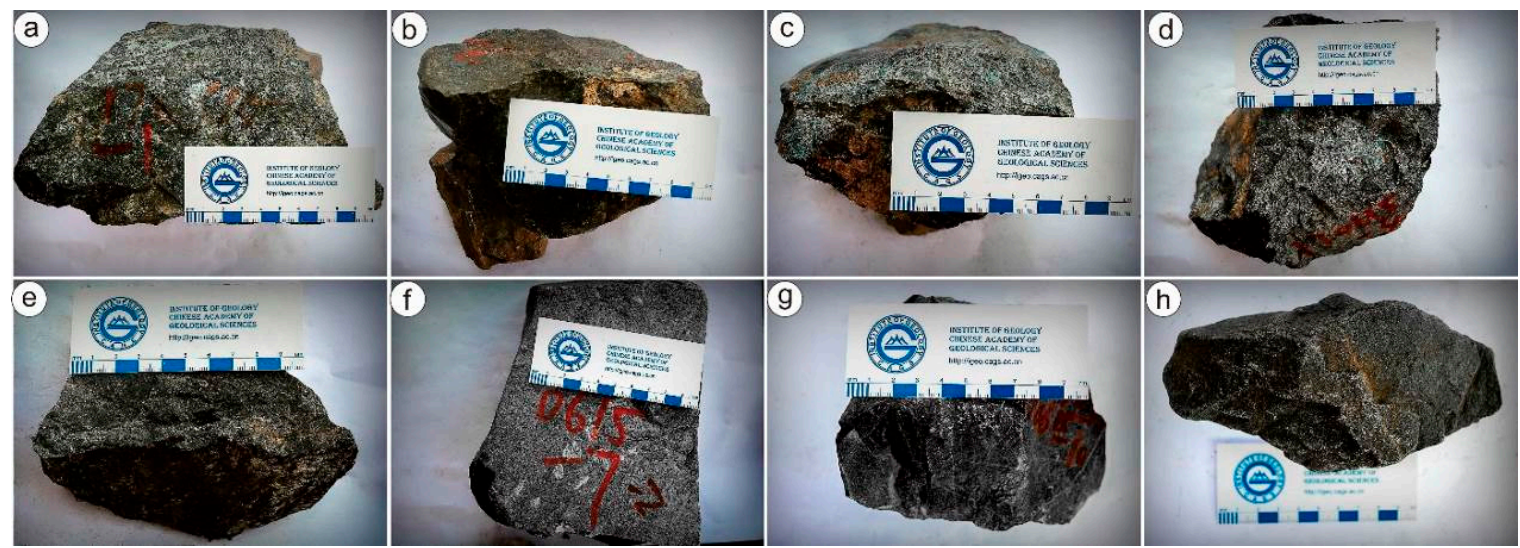

Figure 3. Photographs of the rock samples from the Kaishantun ophiolite suite, Jilin: (a) 170615-1; (b) 170615-2; (c) 170615-3; (d) 170615-4; (e) 170615-6; (f) 170615-7-2; (g) 170615-10; and (h) 170615-11.

Table 1. Sample location, and lithology descriptions; The Kaishantun ophiolite suite, Jilin.

\begin{tabular}{cccc}
\hline Sample No. & Lithology & Location & Description \\
\hline $170615-1$ & peridotite & $42^{\circ} 37^{\prime} 08.57^{\prime \prime} 129^{\circ} 4139.05^{\prime \prime}$ & Yellow or green in color, opaque, massive texture \\
$170615-2$ & peridotite & $42^{\circ} 37^{\prime} 08.57^{\prime \prime} 129^{\circ} 41^{\prime} 39.05^{\prime \prime}$ & Yellow or green in color, opaque, massive texture \\
$170615-3$ & peridotite & $42^{\circ} 37^{\prime} 08.57^{\prime \prime} 129^{\circ} 41^{\prime} 39.05^{\prime \prime}$ & Yellow or green in color, opaque, massive texture \\
$170615-4$ & peridotite & $42^{\circ} 37^{\prime} 08.57^{\prime \prime} 129^{\circ} 41^{\prime} 39.05^{\prime \prime}$ & Yellow or green in color, opaque, massive texture \\
$170615-6$ & diabase & $42^{\circ} 37^{\prime} 13.24^{\prime \prime} 129^{\circ} 41^{\prime} 39.07^{\prime \prime}$ & Gray or green in color, opaque, massive texture \\
$170615-7-2$ & basalt & $42^{\circ} 37^{\prime} 13.24^{\prime \prime} 129^{\circ} 41^{\prime} 39.07^{\prime \prime}$ & Gray in color, opaque, massive texture \\
$170615-10$ & pyroxenite & $42^{\circ} 37^{\prime} 13.24^{\prime \prime} 129^{\circ} 41^{\prime} 39.07^{\prime \prime}$ & Gray or green in color, opaque, massive texture \\
$170615-11$ & gabbro & $42^{\circ} 36^{\prime} 58.16^{\prime \prime} 129^{\circ} 41^{\prime} 39.09^{\prime \prime}$ & Gray in color, opaque, massive texture \\
\hline
\end{tabular}

Samples microstructures and mineral textural relationships were observed with a Leica DMLP Optical Microscope (Leica DMLP GmbH, Wetzlar, Germany) and an FEI Quata-200 Genesis spectrometer (Hillsboro, OR, USA). An EDAX32 scanning electron microscope (SEM, JEOL, Tokyo, Japan) was used to test the element composition of minerals at the Institute of Geology, Chinese Academy of Geological Sciences.

This experiment measured chemical compositions, and discriminated between mineral phases by using energy dispersive spectrometer (EDS, JEOL, Tokyo, Japan). SEM images recorded in backscatter electron mode and were taken in low-vacuum mode with a focus distance of $18 \mathrm{~mm}$, at $20 \mathrm{keV}$ and 0.5 Torr. A microanalysis system, JEOL-5610LV EDSJEOL, Tokyo, Japan, was used to measure the diameter and the phase compositions of a given selection.

The spectra of mineral samples from the Kaishantun ophiolite suite were measured with a TerraSpec spectrometer (Analytical Spectral Devices, Inc., version 6.4, Malvern PANalytical, Boulder, CO, USA) with NKLST-RSIIA image analysis and remote sensing information, located at China RS Geoinformatics Co, Ltd. (Beijng, China) Table 2 contains the technical specifications of the experimental apparatus.

The experimental procedure was based on Labspec 4 in the ViewSpecPro User Manual. The spectrometer was connected to the laptop and a programmed schedule was set up. The RS3 ASD Inc. software package (Malvern PANalytical, Boulder, CO, USA) was used to record and process the data.

The reflectance spectral measurements of rock samples were taken in the laboratory by using an ASD spectrometer with a 350-2500 nm wavelength range. The resolutions are $1.4 \mathrm{~nm}$ at $350-1000 \mathrm{~nm}$; and $2 \mathrm{~nm}$ at $1000-2500 \mathrm{~nm}$. Spectral analysis was measured at 10 random spots on the rock samples 
and then to get averaged representative spectrum of each samples. We measured the samples without a sunlight source using an accessory light and revised the experimental results by testing a white reference, which the RS3 ASD Inc. software automatically calibrated to a reflection coefficient of 1. To avoid the polluting effects of ambient light, the detector was carefully screened after this calibration. We measured six points on each sample to discern the distinctive spectral features that are used to distinguish different minerals.

Table 2. Technical specifications of the ASD Inc. TerraSpec ${ }^{\circledR}$ spectrometer.

\begin{tabular}{cc}
\hline Item & Parameter \\
\hline Spectral Range & 350 to $2500 \mathrm{~nm}$ \\
& $3 \mathrm{~nm} @ 700 \mathrm{~nm}$ \\
Spectral Resolutions & $6 \mathrm{~nm} @ 1400 \mathrm{~nm}$ \\
& $6 \mathrm{~nm} @ 2100 \mathrm{~nm}$ \\
Sampling Intervals & $1.4 \mathrm{~nm}$ between 350 and $1000 \mathrm{~nm}$ \\
& $2 \mathrm{~nm}$ between 1000 and $2500 \mathrm{~nm}$ \\
Signal to Noise Values & $9500 \mathrm{DN} @ 700 \mathrm{~nm}$ \\
& $5000 \mathrm{DN} @ 1400 \mathrm{~nm}$ \\
& $800 \mathrm{DN} @ 2100 \mathrm{~nm}$ \\
\hline
\end{tabular}

The minerals' spectral data were stored in TEXT files by the ViewSpecPro and RS3 software. These TEXT data were converted to Microsoft Excel files and plotted with the CorelDRAW Graphics Suite 2017 (Corel Corporation, Ottawa, ON, Canada). The raw data are included in the Supplementary Materials. Table 3 contains the list of experimental minerals and their spectral file names.

Table 3. File name list of reference and sample spectra.

\begin{tabular}{ccc}
\hline File Name List & Sample No. & Comment \\
\hline White Reference & White Reference & Standard (1 measurement) \\
615-1-1.xls to 615-1-6.xls & $17615-1$ & peridotite (6 measurements) \\
615-2-1.xls to 615-2-6.xls & $17615-2$ & peridotite (6 measurements) \\
615-3-1.xls to 615-3-6.xls & $17615-3$ & peridotite (6 measurements) \\
615-4-1.xls to 615-4-6.xls & $17615-4$ & peridotite (6 measurements) \\
615-6-1.xls to 615-6-6.xls & $17615-6$ & diabase (6 measurements) \\
615-7-2-1.xls to 615-7-2-6.xls & $17615-7-2$ & basalt (6 measurements) \\
615-10-1.xls to 615-10-6.xls & $17615-10$ & pyroxenite (6 measurements) \\
615-11-1.xls to 615-11-6.xls & $17615-11$ & gabbro (6 measurements) \\
\hline
\end{tabular}

Note: Data file in the Supplementary Material.

\section{Results}

\subsection{Petrology}

Eight representative samples from the Kaishantun ophiolite suite were examined in this study for their petrological characteristics. The sample locations are shown in Figure 2, and their salient details are listed in Table 1. The samples exhibited signs of intense tectonic shear deformation and alteration, with corresponding minerals that were mostly subjected to low-grade metamorphism. The samples included peridotite, diabase, basal, pyroxenite, and gabbro. A brief description of the petrographic features of the different rock types is given below, and representative photomicrographs are shown in Figure 4. 

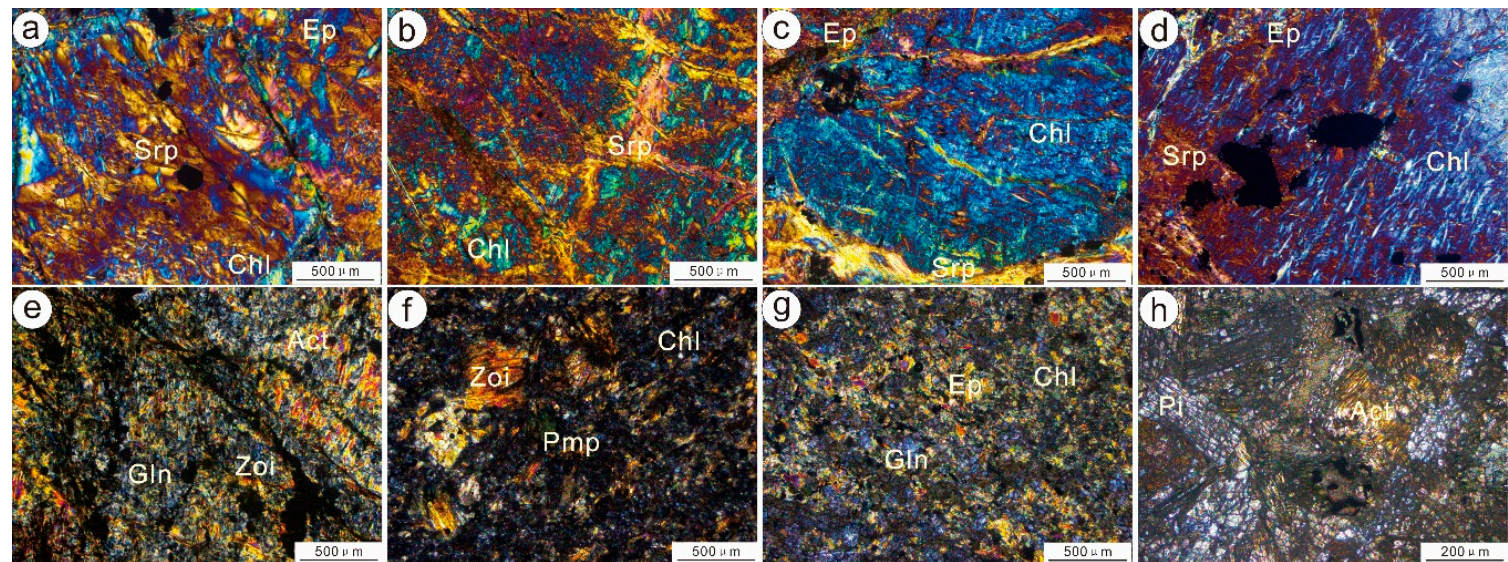

Figure 4. Photomicrographs of minerals and textures in the Kaishantun ophiolite suite. Label as shown in Figure 3: (a) 17615-1; (b) 17615-2; (c) 17615-3; (d) 17615-4; (e) 17615-6; (f) 17615-7-2; (g) 17615-10; and (h) 17615-11. Srp-Serpentine; Ep-Epidote; Chl-Chlorite; Gln-Glaucophane; Zoi-Zoisite;

Pmp-Pumpellyite; Pi-Plagioclase; and Act-Actinolite.

\subsubsection{Serpentinized Peridotite}

Samples 170615-1, 170615-2, 170615-3, and 170615-4 are ultramafic rocks in the Kaishantun ophiolite suite. The hand specimen rocks have pale yellow and light green color, foliated structure and partly altered.

Under the microscope, samples display metasomatic pseudomorph texture, comprising serpentine $(60-70 \%)$, chlorite $(25-35 \%)$, and actinolite $(10-15 \%)$, and the magnetite is also associated with mineral pyroxene (Figure $4 \mathrm{a}-\mathrm{d}$ ). The plagioclase and other felsic minerals are deficiency in the samples with almost no clinopyroxene. The serpentine, which replaced olivine, displays pale yellow color, and is slightly pleochroic, with subhedral and foliated or net-vein texture and flake size of about 0.01 to $0.5 \mathrm{~mm}$ diameter. The mineral contains fine grained spinel inclusions of spinel at the core domains. It is usually surrounded by foliated chlorite, with flake size of about 0.02 to $0.2 \mathrm{~mm}$ diameter and abnormal interference color. The actinolite partly replaced pyroxene, and formed pseudomorph structure, has allotriomorphic fine grain $(<0.1 \mathrm{~mm})$. Magnetite occurs along the grain boundary of actinolite and as the accessory mineral of serpentine-an alteration product.

\subsubsection{Intensely Altered Diabase}

Sample 170615-6 is an altered diabase in the Kaishantun ophiolite suite. The hand specimen rock is dark colored, medium to coarse grained, and blocky structure.

Under the microscope, sample displays blastophitic texture, comprising actynolin (35-45\%), zoisite $(25-35 \%)$, and glaucophane $(10-15 \%)$. There is almost no clinopyroxene. The sample presents metasomatic structure with zoisite-rich and actynolin-rich domain band (Figure 4e).

The actynolin crystals, which replaced clinopyroxene, are subhedral to anhedral, yellowish to greenish, columnar and granular-columnar in shape, ranging in size from 0.2 to $1 \mathrm{~mm}$ and showing non-preferred orientation. The zoisite grains are mostly subhedral, and set in granular or columnar texture with size less than $0.1 \mathrm{~mm}$. The mineral shows obvious pleochroism from pale yellow to light green. The mineral partly assembled subhedral tabular texture and showing non-preferred orientation, as an alteration product replaced plagioclase. The glaucophane displays subhedral to anhedral, granular in shape, and ranging in fine grained diameter from 0.01 to $0.1 \mathrm{~mm}$. 


\subsubsection{Intensely Altered Basalt}

Samples 170615-7-2 is a basic rock in the Kaishantun ophiolite suite. The hand specimen rock is dark grey colored, and blocky structure with part alteration.

Under the microscope, sample displays palimpsest texture, comprising chlorite (40-55\%), zoisite (25-30\%), and minor pumpellyite (5-10\%) (Figure 4f). The rocks are altered. The plagioclase and other felsic minerals are deficiency in the sample with almost no clinopyroxene. The chlorite displays pale or dark green color, and has low interference color, with subhedral and anhedral texture and granular or columnar in shape-grain size less than $0.2 \mathrm{~mm}$ diameter. The zoisite is fine porphyritic-columnar in shape (1-2 $\mathrm{mm})$, subhedral, and mostly present as blastoporphyritic texture. The pumpellyite is subhedral to anhedral, mainly schistose structure with dark green, flake diameter ranging $0.02-0.2 \mathrm{~mm}$. Minerals occurs along the boundary of chlorite with abnormal interference color.

\subsubsection{Intensely Altered Pyroxenite}

Pyroxenite sample 170615-10 is dark colored and compact massive, with epidote (40-50\%), glaucophane (15-25\%) and chlorite (5-10\%) (Figure 4g). The rocks are altered. The plagioclase and other felsic minerals are deficient in the sample with almost no clinopyroxene. Except partly medium grained $(0.5-1 \mathrm{~mm})$, the epidote displays grain size usually less than $0.1 \mathrm{~mm}$, subhedral columnar or granular, and shows light yellow color with little pleochroism, as an alteration product replaced plagioclase. The glaucophane displays subhedral to anhedral, granular in shape, and ranging in fine grained diameter from 0.1 to $0.3 \mathrm{~mm}$. The glaucophane occurs as tabular relics with blue or purple under plane-polarized light. The chlorite mainly occurs along grain boundaries and cleavage traces of the epidote.

\subsubsection{Intensely Altered Gabbro}

Samples 170615-11 were collected from near sample 170615-10. The hand specimen rock is dark grey colored, fine to medium grained, and has foliated structure.

Under the microscope, the rock displays palimpsest gabbroic-ophitic texture composed of plagioclase (40-55\%), pyroxene (30-40\%), and minor actinolite (5-10\%) (Figure $4 \mathrm{~h}$ ).

The plagioclase is sericitization in medium degree with colorless subhedral tabular in shape. Plagioclase displays polygonal distortion and deformation with wavy extinction. Pyroxene shows pyroxene-rich bands, associating with plagioclase. The pyroxenes are well oriented with brown to dark green colored and subhedral granular in these bands. Actinolite grain, which might regard as an alteration pseudomorph of pyroxene, usually occurs interlocked with the plagioclase as a relict crystal. The actinolite is slightly oriented with pale yellow colored, and surround the pyroxene as a dark colored rim.

\subsection{Chemical Compositions}

The mineral testing position and chemical composition characteristics of the Kaishantun ophiolite suite are shown in Figure 5 and Table 4. Ten spots in each sample were measured by an energy dispersive spectrometer (EDS). Compared with standard chemical compositions in "An introduction to the rock-forming minerals" [23], the EDS data show the chemical composition of samples. Formula recalculation was used AX Win 2007 [24]. Mineral within sample 17615-1 displays $\mathrm{Na}_{2} \mathrm{O}$ contents ranges from 0.04 to $0.13 \mathrm{wt} \%$ (average value $0.09 \%$ ). $\mathrm{MgO}$ contents ranges from 35.31 to $43.02 \mathrm{wt} \%$ (average value $37.71 \%$ ). $\mathrm{Al}_{2} \mathrm{O}_{3}$ contents ranges from 1.04 to $2.92 \mathrm{wt} \%$ (average value $1.72 \%$ ). $\mathrm{SiO}_{2}$ contents ranges from 32.48 to $42.07 \mathrm{wt} \%$ (average value $38.27 \%$ ). $\mathrm{MnO}$ contents ranges from 0.02 to $0.82 \mathrm{wt} \%$ (average value $0.45 \%$ ). $\mathrm{FeO}_{\mathrm{T}}$ and $\mathrm{Cr}_{2} \mathrm{O}_{3}$ contents range from 4.09 to $6.87 \mathrm{wt} \%$ (average value $5.21 \%$ ) and from 0.13 to $0.97 \mathrm{wt} \%$ (average value $0.54 \%$ ), respectively. The chemical compositions are close to common serpentine with an approximate mineral formula that can be inferred as $\mathrm{Mg}_{3} \mathrm{Si}_{2} \mathrm{O}_{5}(\mathrm{OH})_{\mathrm{X}}$ [23]. 


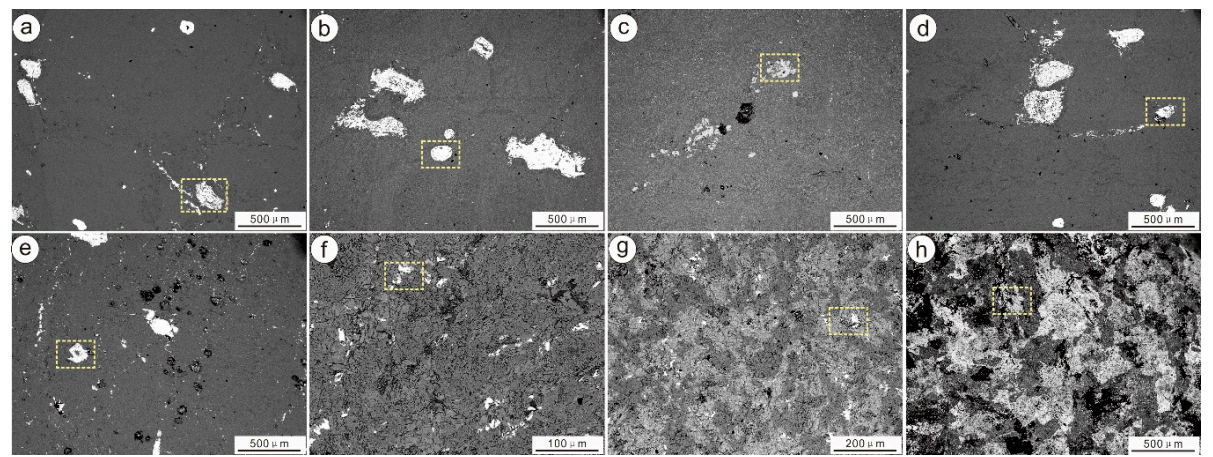

Figure 5. SEM back-scattered electron images of section samples in Kaishantun ophiolite suite: (a) 170615-1; (b) 170615-2; (c) 170615-3; (d) 170615-4; (e) 170615-6; (f) 170615-7-2; (g) 170615-10; and (h) 170615-11. Label as shown in Figure 3. Rectangular box is the testing position, ten spots in each sample were measured in these areas.

Mineral within sample 17615-2 shows similar chemical compositions with 17615-1: $\mathrm{Na}_{2} \mathrm{O}$ contents ranges from 0.01 to $0.15 \mathrm{wt} \%$ (average value $0.07 \%$ ). $\mathrm{MgO}$ contents ranges from 35.31 to $43.02 \mathrm{wt} \%$ (average value 37.41\%). $\mathrm{Al}_{2} \mathrm{O}_{3}$ contents ranges from 0.47 to $4.23 \mathrm{wt} \%$ (average value $1.61 \%$ ). $\mathrm{SiO}_{2}$ contents ranges from 39.53 to $52.93 \mathrm{wt} \%$ (average value $45.37 \%$ ). $\mathrm{TiO}_{2}$ contents ranges from 0.18 to $0.86 \mathrm{wt} \%$ (average value $0.54 \%$ ). $\mathrm{MnO}$ and $\mathrm{FeO}_{\mathrm{T}}$ contents range from 0.03 to $0.26 \mathrm{wt} \%$ (average value $0.10 \%$ ) and from 4.97 to $8.28 \mathrm{wt} \%$ (average value $6.26 \%$ ). It is also close to the chemical compositions of common serpentine with an approximate mineral formula that can be inferred as $\mathrm{Mg}_{3} \mathrm{Si}_{2} \mathrm{O}_{5}(\mathrm{OH})_{X}[23]$.

Table 4. Chemical compositions of mineral samples in representative rocks from Kaishantun ophiolite. (wt \%).

\begin{tabular}{ccccccccccc}
\hline Sample & & \multicolumn{10}{c}{$\mathbf{1 7 6 1 5 - 1}$} \\
\hline Mineral & & & \multicolumn{9}{c}{ Serpentine } \\
\hline Positions & $\mathbf{1}$ & $\mathbf{2}$ & $\mathbf{3}$ & $\mathbf{4}$ & $\mathbf{5}$ & $\mathbf{6}$ & $\mathbf{7}$ & $\mathbf{8}$ & $\mathbf{9}$ & $\mathbf{1 0}$ \\
\hline $\mathrm{Na}_{2} \mathrm{O}$ & 0.12 & 0.06 & 0.04 & 0.08 & 0.13 & 0.07 & 0.05 & 0.11 & 0.07 & 0.13 \\
$\mathrm{MgO}$ & 38.22 & 37.84 & 35.72 & 35.55 & 36.79 & 35.31 & 37.42 & 39.22 & 38.01 & 43.02 \\
$\mathrm{Al}_{2} \mathrm{O}_{3}$ & 1.89 & 2.92 & 1.70 & 1.93 & 1.08 & 1.47 & 1.04 & 1.71 & 1.59 & 1.86 \\
$\mathrm{SiO}_{2}$ & 42.07 & 38.09 & 40.74 & 32.48 & 38.91 & 34.25 & 39.31 & 39.79 & 38.26 & 38.84 \\
$\mathrm{~K}_{2} \mathrm{O}$ & 0.00 & 0.00 & 0.00 & 0.00 & 0.00 & 0.00 & 0.00 & 0.00 & 0.00 & 0.00 \\
$\mathrm{CaO}$ & 0.00 & 0.00 & 0.00 & 0.00 & 0.00 & 0.00 & 0.00 & 0.00 & 0.00 & 0.00 \\
$\mathrm{TiO}_{2}$ & 0.00 & 0.00 & 0.00 & 0.00 & 0.00 & 0.00 & 0.00 & 0.00 & 0.00 & 0.00 \\
$\mathrm{MnO}$ & 0.76 & 0.82 & 0.02 & 0.38 & 0.12 & 0.41 & 0.40 & 0.59 & 0.52 & 0.47 \\
$\mathrm{FeO}$ & 5.22 & 4.09 & 4.69 & 5.13 & 4.97 & 6.87 & 5.28 & 5.72 & 4.23 & 5.90 \\
$\mathrm{Cr}_{2} \mathrm{O}_{3}$ & 0.51 & 0.24 & 0.13 & 0.54 & 0.27 & 0.40 & 0.76 & 0.97 & 0.67 & 0.91 \\
$\mathrm{Total}$ & 88.79 & 84.06 & 83.04 & 76.09 & 82.27 & 78.78 & 84.26 & 88.11 & 83.35 & 91.13 \\
$\mathrm{Si}$ & 1.121 & 1.071 & 1.149 & 1.026 & 1.118 & 1.051 & 1.108 & 1.078 & 1.087 & 1.025 \\
$\mathrm{Ti}$ & 0.000 & 0.000 & 0.000 & 0.000 & 0.000 & 0.000 & 0.000 & 0.000 & 0.000 & 0.000 \\
$\mathrm{Al}$ & 0.059 & 0.097 & 0.057 & 0.072 & 0.037 & 0.053 & 0.035 & 0.055 & 0.053 & 0.058 \\
$\mathrm{Cr}$ & 0.011 & 0.005 & 0.003 & 0.013 & 0.006 & 0.010 & 0.017 & 0.021 & 0.015 & 0.019 \\
$\mathrm{Fe}{ }^{3+}$ & 0.000 & 0.000 & 0.000 & 0.000 & 0.000 & 0.000 & 0.000 & 0.000 & 0.000 & 0.000 \\
$\mathrm{Fe}{ }^{2+}$ & 0.116 & 0.096 & 0.111 & 0.135 & 0.119 & 0.176 & 0.124 & 0.130 & 0.100 & 0.130 \\
$\mathrm{Mn}$ & 0.017 & 0.020 & 0.000 & 0.010 & 0.003 & 0.011 & 0.010 & 0.014 & 0.013 & 0.011 \\
$\mathrm{Mg}$ & 1.517 & 1.586 & 1.501 & 1.673 & 1.575 & 1.615 & 1.572 & 1.584 & 1.609 & 1.691 \\
$\mathrm{Ca}$ & 0.000 & 0.000 & 0.000 & 0.000 & 0.000 & 0.000 & 0.000 & 0.000 & 0.000 & 0.000 \\
$\mathrm{Na}$ & 0.006 & 0.003 & 0.002 & 0.005 & 0.007 & 0.004 & 0.003 & 0.006 & 0.004 & 0.007 \\
$\mathrm{~K}$ & 0.000 & 0.000 & 0.000 & 0.000 & 0.000 & 0.000 & 0.000 & 0.000 & 0.000 & 0.000 \\
$\mathrm{Sum}$ & 2.847 & 2.879 & 2.823 & 2.934 & 2.865 & 2.920 & 2.868 & 2.887 & 2.881 & 2.940 \\
\hline
\end{tabular}


Table 4. Cont.

\begin{tabular}{|c|c|c|c|c|c|c|c|c|c|c|}
\hline \multirow{3}{*}{$\begin{array}{c}\text { Sample } \\
\text { Mineral } \\
\text { Positions } \\
\end{array}$} & \multicolumn{10}{|c|}{ 17615-2 } \\
\hline & \multicolumn{10}{|c|}{ Serpentine } \\
\hline & 1 & 2 & 3 & 4 & 5 & 6 & 7 & 8 & 9 & 10 \\
\hline $\mathrm{Na}_{2} \mathrm{O}$ & 0.10 & 0.14 & 0.09 & 0.03 & 0.15 & 0.04 & 0.05 & 0.01 & 0.05 & 0.02 \\
\hline $\mathrm{MgO}$ & 35.79 & 36.10 & 37.52 & 37.71 & 42.54 & 39.37 & 34.03 & 36.95 & 38.46 & 35.66 \\
\hline $\mathrm{Al}_{2} \mathrm{O}_{3}$ & 4.21 & 2.38 & 4.23 & 0.47 & 0.95 & 0.61 & 0.32 & 1.85 & 0.54 & 0.56 \\
\hline $\mathrm{SiO}_{2}$ & 42.89 & 46.16 & 39.53 & 45.52 & 47.28 & 43.47 & 48.51 & 43.10 & 44.27 & 52.93 \\
\hline $\mathrm{K}_{2} \mathrm{O}$ & 0.00 & 0.00 & 0.00 & 0.00 & 0.00 & 0.00 & 0.00 & 0.00 & 0.00 & 0.00 \\
\hline $\mathrm{CaO}$ & 0.00 & 0.00 & 0.00 & 0.00 & 0.00 & 0.00 & 0.00 & 0.00 & 0.00 & 0.00 \\
\hline $\mathrm{TiO}_{2}$ & 0.33 & 0.39 & 0.54 & 0.69 & 0.18 & 0.61 & 0.68 & 0.86 & 0.45 & 0.70 \\
\hline $\mathrm{MnO}$ & 0.07 & 0.26 & 0.15 & 0.11 & 0.09 & 0.03 & 0.05 & 0.06 & 0.11 & 0.08 \\
\hline $\mathrm{FeO}_{\mathrm{T}}$ & 5.77 & 4.97 & 5.17 & 5.61 & 7.06 & 5.92 & 7.45 & 5.24 & 8.28 & 7.15 \\
\hline $\mathrm{Cr}_{2} \mathrm{O}_{3}$ & 0.00 & 0.00 & 0.00 & 0.00 & 0.00 & 0.00 & 0.00 & 0.00 & 0.00 & 0.00 \\
\hline Total & 89.16 & 90.40 & 87.23 & 90.14 & 98.25 & 90.05 & 91.09 & 88.07 & 92.16 & 97.10 \\
\hline $\mathrm{Si}$ & 1.128 & 1.188 & 1.070 & 1.183 & 1.139 & 1.139 & 1.247 & 1.147 & 1.146 & 1.267 \\
\hline $\mathrm{Ti}$ & 0.007 & 0.008 & 0.011 & 0.013 & 0.003 & 0.012 & 0.013 & 0.017 & 0.009 & 0.013 \\
\hline $\mathrm{Al}$ & 0.131 & 0.072 & 0.135 & 0.014 & 0.027 & 0.019 & 0.010 & 0.058 & 0.016 & 0.016 \\
\hline $\mathrm{Cr}$ & 0.000 & 0.000 & 0.000 & 0.000 & 0.000 & 0.000 & 0.000 & 0.000 & 0.000 & 0.000 \\
\hline $\mathrm{Fe}^{3+}$ & 0.000 & 0.000 & 0.000 & 0.000 & 0.000 & 0.000 & 0.000 & 0.000 & 0.000 & 0.000 \\
\hline $\mathrm{Fe}^{2+}$ & 0.127 & 0.107 & 0.117 & 0.122 & 0.142 & 0.130 & 0.160 & 0.117 & 0.179 & 0.143 \\
\hline $\mathrm{Mn}$ & 0.002 & 0.006 & 0.003 & 0.002 & 0.002 & 0.001 & 0.001 & 0.001 & 0.002 & 0.002 \\
\hline $\mathrm{Mg}$ & 1.403 & 1.385 & 1.513 & 1.460 & 1.527 & 1.538 & 1.303 & 1.466 & 1.483 & 1.272 \\
\hline $\mathrm{Ca}$ & 0.000 & 0.000 & 0.000 & 0.000 & 0.000 & 0.000 & 0.000 & 0.000 & 0.000 & 0.000 \\
\hline $\mathrm{Na}$ & 0.005 & 0.007 & 0.005 & 0.002 & 0.007 & 0.002 & 0.002 & 0.001 & 0.003 & 0.001 \\
\hline K & 0.000 & 0.000 & 0.000 & 0.000 & 0.000 & 0.000 & 0.000 & 0.000 & 0.000 & 0.000 \\
\hline Sum & 2.802 & 2.772 & 2.854 & 2.797 & 2.848 & 2.840 & 2.737 & 2.807 & 2.839 & 2.713 \\
\hline Sample & \multicolumn{10}{|c|}{ 17615-3 } \\
\hline Mineral & \multicolumn{10}{|c|}{ Epidote } \\
\hline Positions & 1 & 2 & 3 & 4 & 5 & 6 & 7 & 8 & 9 & 10 \\
\hline $\mathrm{Na}_{2} \mathrm{O}$ & 0.00 & 0.00 & 0.00 & 0.00 & 0.00 & 0.00 & 0.00 & 0.00 & 0.00 & 0.00 \\
\hline $\mathrm{MgO}$ & 0.03 & 0.64 & 0.54 & 0.37 & 0.28 & 0.93 & 0.79 & 0.81 & 0.14 & 0.50 \\
\hline $\mathrm{Al}_{2} \mathrm{O}_{3}$ & 18.89 & 21.64 & 20.78 & 22.79 & 23.29 & 21.88 & 19.71 & 22.10 & 21.05 & 18.95 \\
\hline $\mathrm{SiO}_{2}$ & 33.10 & 34.36 & 35.01 & 31.09 & 32.51 & 32.09 & 34.72 & 32.84 & 35.51 & 35.20 \\
\hline $\mathrm{K}_{2} \mathrm{O}$ & 0.00 & 0.00 & 0.00 & 0.00 & 0.00 & 0.00 & 0.00 & 0.00 & 0.00 & 0.00 \\
\hline $\mathrm{CaO}$ & 26.17 & 25.07 & 23.25 & 24.96 & 27.54 & 23.09 & 23.59 & 25.81 & 23.49 & 24.19 \\
\hline $\mathrm{TiO}_{2}$ & 0.00 & 0.00 & 0.00 & 0.00 & 0.00 & 0.00 & 0.00 & 0.00 & 0.00 & 0.00 \\
\hline $\mathrm{MnO}$ & 0.00 & 0.00 & 0.00 & 0.00 & 0.00 & 0.00 & 0.00 & 0.00 & 0.00 & 0.00 \\
\hline $\mathrm{FeO}_{\mathrm{T}}$ & 8.81 & 9.28 & 10.41 & 9.78 & 8.37 & 9.04 & 10.20 & 9.45 & 11.81 & 9.15 \\
\hline $\mathrm{Cr}_{2} \mathrm{O}_{3}$ & 0.00 & 0.00 & 0.00 & 0.00 & 0.00 & 0.00 & 0.00 & 0.00 & 0.00 & 0.00 \\
\hline Total & 87.00 & 90.99 & 89.99 & 88.99 & 91.99 & 87.03 & 89.01 & 91.01 & 92.00 & 87.99 \\
\hline $\mathrm{Si}$ & 3.063 & 3.013 & 3.079 & 2.817 & 2.839 & 2.937 & 3.116 & 2.900 & 3.035 & 3.183 \\
\hline $\mathrm{Ti}$ & 0.000 & 0.000 & 0.000 & 0.000 & 0.000 & 0.000 & 0.000 & 0.000 & 0.000 & 0.000 \\
\hline $\mathrm{Al}$ & 2.061 & 2.237 & 2.155 & 2.434 & 2.398 & 2.361 & 2.085 & 2.301 & 2.121 & 2.020 \\
\hline $\mathrm{Cr}$ & 0.000 & 0.000 & 0.000 & 0.000 & 0.000 & 0.000 & 0.000 & 0.000 & 0.000 & 0.000 \\
\hline $\mathrm{Fe}^{3+}$ & 0.000 & 0.000 & 0.162 & 0.000 & 0.000 & 0.000 & 0.000 & 0.000 & 0.471 & 0.000 \\
\hline $\mathrm{Fe}^{2+}$ & 0.682 & 0.680 & 0.603 & 0.741 & 0.611 & 0.692 & 0.766 & 0.698 & 0.373 & 0.692 \\
\hline $\mathrm{Mn}$ & 0.000 & 0.000 & 0.000 & 0.000 & 0.000 & 0.000 & 0.000 & 0.000 & 0.000 & 0.000 \\
\hline $\mathrm{Mg}$ & 0.004 & 0.084 & 0.071 & 0.050 & 0.036 & 0.127 & 0.106 & 0.107 & 0.018 & 0.067 \\
\hline $\mathrm{Ca}$ & 2.595 & 2.355 & 2.191 & 2.423 & 2.577 & 2.265 & 2.269 & 2.443 & 2.151 & 2.344 \\
\hline $\mathrm{Na}$ & 0.000 & 0.000 & 0.000 & 0.000 & 0.000 & 0.000 & 0.000 & 0.000 & 0.000 & 0.000 \\
\hline $\mathrm{K}$ & 0.000 & 0.000 & 0.000 & 0.000 & 0.000 & 0.000 & 0.000 & 0.000 & 0.000 & 0.000 \\
\hline Sum & 8.407 & 8.370 & 8.264 & 8.467 & 8.463 & 8.383 & 8.342 & 8.450 & 8.171 & 8.308 \\
\hline
\end{tabular}


Table 4. Cont

\begin{tabular}{|c|c|c|c|c|c|c|c|c|c|c|}
\hline Sample & & & & & 176 & $5-4$ & & & & \\
\hline Mineral & & & & & Acti & olite & & & & \\
\hline Positions & 1 & 2 & 3 & 4 & 5 & 6 & 7 & 8 & 9 & 10 \\
\hline $\mathrm{Na}_{2} \mathrm{O}$ & 0.00 & 0.00 & 0.00 & 0.00 & 0.00 & 0.00 & 0.00 & 0.00 & 0.00 & 0.00 \\
\hline $\mathrm{MgO}$ & 10.57 & 14.16 & 13.14 & 16.97 & 13.64 & 14.90 & 11.92 & 12.21 & 13.23 & 13.92 \\
\hline $\mathrm{Al}_{2} \mathrm{O}_{3}$ & 4.73 & 9.82 & 6.38 & 3.21 & 5.09 & 4.57 & 8.66 & 8.01 & 6.39 & 5.48 \\
\hline $\mathrm{SiO}_{2}$ & 49.85 & 49.76 & 47.95 & 49.04 & 51.03 & 45.53 & 46.42 & 51.08 & 52.71 & 55.85 \\
\hline $\mathrm{K}_{2} \mathrm{O}$ & 0.00 & 0.00 & 0.00 & 0.00 & 0.00 & 0.00 & 0.00 & 0.00 & 0.00 & 0.00 \\
\hline $\mathrm{CaO}$ & 9.31 & 9.47 & 10.71 & 9.29 & 10.72 & 11.84 & 11.54 & 9.94 & 10.13 & 11.48 \\
\hline $\mathrm{TiO}_{2}$ & 0.00 & 0.00 & 0.00 & 0.00 & 0.00 & 0.00 & 0.00 & 0.00 & 0.00 & 0.00 \\
\hline $\mathrm{MnO}$ & 0.00 & 0.00 & 0.00 & 0.00 & 0.00 & 0.00 & 0.00 & 0.00 & 0.00 & 0.00 \\
\hline $\mathrm{FeO}_{\mathrm{T}}$ & 18.65 & 13.79 & 17.03 & 15.49 & 16.53 & 15.18 & 13.46 & 13.76 & 14.55 & 12.28 \\
\hline $\mathrm{Cr}_{2} \mathrm{O}_{3}$ & 0.00 & 0.00 & 0.00 & 0.00 & 0.00 & 0.00 & 0.00 & 0.00 & 0.00 & 0.00 \\
\hline Total & 93.11 & 97.00 & 95.21 & 94.00 & 97.01 & 92.02 & 92.00 & 95.00 & 97.01 & 99.01 \\
\hline $\mathrm{Si}$ & 7.68 & 7.17 & 7.14 & 7.42 & 7.46 & 7.14 & 7.10 & 7.50 & 7.60 & 7.80 \\
\hline $\mathrm{Ti}$ & 0.000 & 0.000 & 0.000 & 0.000 & 0.000 & 0.000 & 0.000 & 0.000 & 0.000 & 0.000 \\
\hline $\mathrm{Al}$ & 0.859 & 1.668 & 1.120 & 0.573 & 0.877 & 0.845 & 1.562 & 1.386 & 1.087 & 0.902 \\
\hline $\mathrm{Cr}$ & 0.000 & 0.000 & 0.000 & 0.000 & 0.000 & 0.000 & 0.000 & 0.000 & 0.000 & 0.000 \\
\hline $\mathrm{Fe}^{3+}$ & 0.227 & 0.269 & 0.146 & 0.139 & 0.161 & 0.034 & 0.054 & 0.208 & 0.210 & 0.127 \\
\hline $\mathrm{Fe}^{2+}$ & 2.174 & 1.392 & 1.368 & 1.822 & 1.646 & 1.956 & 1.439 & 1.481 & 1.545 & 1.307 \\
\hline $\mathrm{Mn}$ & 0.000 & 0.000 & 0.000 & 0.000 & 0.000 & 0.000 & 0.000 & 0.000 & 0.000 & 0.000 \\
\hline $\mathrm{Mg}$ & 2.425 & 3.040 & 2.915 & 3.828 & 2.970 & 3.481 & 2.719 & 2.671 & 2.844 & 2.897 \\
\hline $\mathrm{Ca}$ & 1.536 & 1.462 & 1.708 & 1.507 & 1.678 & 1.989 & 1.892 & 1.563 & 1.566 & 1.718 \\
\hline $\mathrm{Na}$ & 0.000 & 0.000 & 0.000 & 0.000 & 0.000 & 0.000 & 0.000 & 0.000 & 0.000 & 0.000 \\
\hline K & 0.000 & 0.000 & 0.000 & 0.000 & 0.000 & 0.000 & 0.000 & 0.000 & 0.000 & 0.000 \\
\hline Sum & 14.896 & 14.999 & 15.201 & 15.291 & 15.070 & 15.442 & 15.076 & 14.809 & 14.855 & 14.751 \\
\hline Sample & & & & & 176 & & & & & \\
\hline Mineral & & & & & Glauc & phane & & & & \\
\hline Positions & 1 & 2 & 3 & 4 & 5 & 6 & 7 & 8 & 9 & 10 \\
\hline $\mathrm{Na}_{2} \mathrm{O}$ & 6.97 & 2.40 & 1.45 & 6.18 & 4.46 & 5.29 & 7.23 & 1.14 & 4.31 & 2.13 \\
\hline $\mathrm{MgO}$ & 12.97 & 12.51 & 14.54 & 12.42 & 11.75 & 9.86 & 9.30 & 8.16 & 9.31 & 8.30 \\
\hline $\mathrm{Al}_{2} \mathrm{O}_{3}$ & 11.19 & 12.82 & 10.01 & 10.98 & 10.93 & 13.71 & 11.26 & 10.95 & 10.79 & 9.52 \\
\hline $\mathrm{SiO}_{2}$ & 49.29 & 52.00 & 52.51 & 51.88 & 48.25 & 52.32 & 54.85 & 49.08 & 49.94 & 51.42 \\
\hline $\mathrm{K}_{2} \mathrm{O}$ & 0.00 & 0.00 & 0.00 & 0.00 & 0.00 & 0.00 & 0.00 & 0.00 & 0.00 & 0.00 \\
\hline $\mathrm{CaO}$ & 1.41 & 8.16 & 9.72 & 5.95 & 5.07 & 6.60 & 2.14 & 8.76 & 4.69 & 5.28 \\
\hline $\mathrm{TiO}_{2}$ & 0.20 & 0.66 & 0.42 & 0.05 & 0.09 & 0.71 & 1.36 & 2.96 & 0.53 & 0.90 \\
\hline $\mathrm{MnO}$ & 0.00 & 0.00 & 0.00 & 0.00 & 0.00 & 0.00 & 0.00 & 0.00 & 0.00 & 0.00 \\
\hline $\mathrm{FeO}_{\mathrm{T}}$ & 13.87 & 11.44 & 10.35 & 12.49 & 13.34 & 10.52 & 13.85 & 15.96 & 19.43 & 19.46 \\
\hline $\mathrm{Cr}_{2} \mathrm{O}_{3}$ & 0.00 & 0.00 & 0.00 & 0.00 & 0.00 & 0.00 & 0.00 & 0.00 & 0.00 & 0.00 \\
\hline Total & 95.90 & 99.99 & 99.00 & 99.95 & 93.89 & 99.01 & 99.99 & 97.01 & 99.00 & 97.01 \\
\hline $\mathrm{Si}$ & 7.096 & 7.153 & 7.289 & 7.220 & 7.102 & 7.265 & 7.560 & 7.146 & 7.134 & 7.514 \\
\hline $\mathrm{Ti}$ & 0.022 & 0.068 & 0.044 & 0.005 & 0.010 & 0.074 & 0.141 & 0.324 & 0.057 & 0.099 \\
\hline $\mathrm{Al}$ & 1.899 & 2.079 & 1.638 & 1.801 & 1.897 & 2.244 & 1.830 & 1.879 & 1.817 & 1.640 \\
\hline $\mathrm{Cr}$ & 0.000 & 0.000 & 0.000 & 0.000 & 0.000 & 0.000 & 0.000 & 0.000 & 0.000 & 0.000 \\
\hline $\mathrm{Fe}^{3+}$ & 0.546 & 0.072 & 0.052 & 0.195 & 0.550 & 0.000 & 0.130 & 0.000 & 0.551 & 0.000 \\
\hline $\mathrm{Fe}^{2+}$ & 1.124 & 1.244 & 1.150 & 1.258 & 1.092 & 1.222 & 1.466 & 1.943 & 1.770 & 2.378 \\
\hline $\mathrm{Mn}$ & 0.000 & 0.000 & 0.000 & 0.000 & 0.000 & 0.000 & 0.000 & 0.000 & 0.000 & 0.000 \\
\hline $\mathrm{Mg}$ & 2.783 & 2.565 & 3.008 & 2.576 & 2.578 & 2.040 & 1.910 & 1.771 & 1.982 & 1.808 \\
\hline $\mathrm{Ca}$ & 0.218 & 1.203 & 1.446 & 0.887 & 0.800 & 0.982 & 0.316 & 1.367 & 0.718 & 0.827 \\
\hline $\mathrm{Na}$ & 1.946 & 0.640 & 0.390 & 1.668 & 1.273 & 1.424 & 1.932 & 0.322 & 1.194 & 0.604 \\
\hline $\mathrm{K}$ & 0.000 & 0.000 & 0.000 & 0.000 & 0.000 & 0.000 & 0.000 & 0.000 & 0.000 & 0.000 \\
\hline Sum & 15.822 & 15.047 & 15.034 & 15.678 & 15.487 & 15.252 & 15.330 & 14.750 & 15.408 & 14.869 \\
\hline
\end{tabular}


Table 4. Cont.

\begin{tabular}{|c|c|c|c|c|c|c|c|c|c|c|}
\hline Sample & & & & & 1761 & $-7-2$ & & & & \\
\hline Mineral & & & & & Zo & & & & & \\
\hline Positions & 1 & 2 & 3 & 4 & 5 & 6 & 7 & 8 & 9 & 10 \\
\hline $\mathrm{Na}_{2} \mathrm{O}$ & 0.00 & 0.00 & 0.00 & 0.00 & 0.00 & 0.00 & 0.00 & 0.00 & 0.00 & 0.00 \\
\hline $\mathrm{MgO}$ & 0.00 & 0.00 & 0.00 & 0.00 & 0.00 & 0.00 & 0.00 & 0.00 & 0.00 & 0.00 \\
\hline $\mathrm{Al}_{2} \mathrm{O}_{3}$ & 31.19 & 32.71 & 37.76 & 32.63 & 36.65 & 35.56 & 33.67 & 35.31 & 37.96 & 37.11 \\
\hline $\mathrm{SiO}_{2}$ & 40.80 & 40.41 & 39.51 & 40.08 & 40.55 & 39.98 & 40.91 & 40.81 & 39.88 & 39.49 \\
\hline $\mathrm{K}_{2} \mathrm{O}$ & 0.00 & 0.00 & 0.00 & 0.00 & 0.00 & 0.00 & 0.00 & 0.00 & 0.00 & 0.00 \\
\hline $\mathrm{CaO}$ & 25.73 & 23.85 & 21.40 & 22.64 & 21.42 & 21.13 & 21.54 & 21.20 & 21.14 & 20.81 \\
\hline $\mathrm{TiO}_{2}$ & 0.00 & 0.00 & 0.00 & 0.00 & 0.00 & 0.00 & 0.00 & 0.00 & 0.00 & 0.00 \\
\hline $\mathrm{MnO}$ & 0.00 & 0.00 & 0.00 & 0.00 & 0.00 & 0.00 & 0.00 & 0.00 & 0.00 & 0.00 \\
\hline $\mathrm{FeO}_{\mathrm{T}}$ & 0.28 & 0.03 & 0.34 & 0.65 & 0.38 & 0.33 & 0.08 & 0.51 & 0.02 & 0.09 \\
\hline $\mathrm{Cr}_{2} \mathrm{O}_{3}$ & 0.00 & 0.00 & 0.00 & 0.00 & 0.00 & 0.00 & 0.00 & 0.00 & 0.00 & 0.00 \\
\hline Total & 98.00 & 97.00 & 99.01 & 96.00 & 99.00 & 97.00 & 96.20 & 97.83 & 99.00 & 97.50 \\
\hline $\mathrm{Si}$ & 3.10 & 3.08 & 2.92 & 3.08 & 2.99 & 3.01 & 3.11 & 3.05 & 2.94 & 2.96 \\
\hline $\mathrm{Ti}$ & 0.000 & 0.000 & 0.000 & 0.000 & 0.000 & 0.000 & 0.000 & 0.000 & 0.000 & 0.000 \\
\hline $\mathrm{Al}$ & 2.793 & 2.935 & 3.290 & 2.951 & 3.189 & 3.159 & 3.016 & 3.108 & 3.299 & 3.274 \\
\hline $\mathrm{Cr}$ & 0.000 & 0.000 & 0.000 & 0.000 & 0.000 & 0.000 & 0.000 & 0.000 & 0.000 & 0.000 \\
\hline $\mathrm{Fe}^{3+}$ & 0.000 & 0.002 & 0.021 & 0.041 & 0.023 & 0.021 & 0.005 & 0.032 & 0.001 & 0.006 \\
\hline $\mathrm{Fe}^{2+}$ & 0.018 & 0.000 & 0.000 & 0.000 & 0.000 & 0.000 & 0.000 & 0.000 & 0.000 & 0.000 \\
\hline $\mathrm{Mn}$ & 0.000 & 0.000 & 0.000 & 0.000 & 0.000 & 0.000 & 0.000 & 0.000 & 0.000 & 0.000 \\
\hline $\mathrm{Mg}$ & 0.000 & 0.000 & 0.000 & 0.000 & 0.000 & 0.000 & 0.000 & 0.000 & 0.000 & 0.000 \\
\hline $\mathrm{Ca}$ & 2.094 & 1.945 & 1.695 & 1.861 & 1.694 & 1.706 & 1.753 & 1.696 & 1.670 & 1.669 \\
\hline $\mathrm{Na}$ & 0.000 & 0.000 & 0.000 & 0.000 & 0.000 & 0.000 & 0.000 & 0.000 & 0.000 & 0.000 \\
\hline K & 0.000 & 0.000 & 0.000 & 0.000 & 0.000 & 0.000 & 0.000 & 0.000 & 0.000 & 0.000 \\
\hline Sum & 8.005 & 7.957 & 7.926 & 7.930 & 7.901 & 7.899 & 7.882 & 7.884 & 7.911 & 7.905 \\
\hline Sample & & & & & 176 & -10 & & & & \\
\hline Mineral & & & & & Chl & rite & & & & \\
\hline Positions & 1 & 2 & 3 & 4 & 5 & 6 & 7 & 8 & 9 & 10 \\
\hline $\mathrm{Na}_{2} \mathrm{O}$ & 0.00 & 0.00 & 0.00 & 0.00 & 0.00 & 0.00 & 0.00 & 0.00 & 0.00 & 0.00 \\
\hline $\mathrm{MgO}$ & 9.21 & 12.38 & 11.23 & 10.47 & 11.95 & 10.61 & 10.32 & 11.85 & 10.54 & 10.56 \\
\hline $\mathrm{Al}_{2} \mathrm{O}_{3}$ & 25.79 & 24.10 & 27.52 & 23.71 & 24.54 & 22.37 & 24.03 & 20.95 & 27.46 & 29.66 \\
\hline $\mathrm{SiO}_{2}$ & 22.89 & 25.16 & 29.53 & 28.52 & 27.28 & 23.47 & 28.51 & 23.10 & 24.27 & 22.93 \\
\hline $\mathrm{K}_{2} \mathrm{O}$ & 0.00 & 0.00 & 0.00 & 0.00 & 0.00 & 0.00 & 0.00 & 0.00 & 0.00 & 0.00 \\
\hline $\mathrm{CaO}$ & 5.33 & 4.39 & 6.54 & 9.69 & 9.18 & 7.61 & 5.68 & 8.86 & 4.45 & 5.70 \\
\hline $\mathrm{TiO}_{2}$ & 0.00 & 0.00 & 0.00 & 0.00 & 0.00 & 0.00 & 0.00 & 0.00 & 0.00 & 0.00 \\
\hline $\mathrm{MnO}$ & 0.00 & 0.00 & 0.00 & 0.00 & 0.00 & 0.00 & 0.00 & 0.00 & 0.00 & 0.00 \\
\hline $\mathrm{FeO}_{\mathrm{T}}$ & 25.77 & 22.97 & 23.17 & 26.61 & 27.06 & 20.92 & 27.45 & 24.24 & 27.28 & 26.15 \\
\hline $\mathrm{Cr}_{2} \mathrm{O}_{3}$ & 0.00 & 0.00 & 0.00 & 0.00 & 0.00 & 0.00 & 0.00 & 0.00 & 0.00 & 0.00 \\
\hline Total & 88.99 & 89.00 & 97.99 & 99.00 & 100.01 & 84.98 & 95.99 & 89.00 & 94.00 & 95.00 \\
\hline $\mathrm{Si}$ & 3.60 & 3.39 & 3.61 & 3.37 & 3.25 & 3.32 & 3.32 & 3.08 & 3.09 & 3.07 \\
\hline $\mathrm{Ti}$ & 0.000 & 0.000 & 0.000 & 0.000 & 0.000 & 0.000 & 0.000 & 0.000 & 0.000 & 0.000 \\
\hline $\mathrm{Al}$ & 0.000 & 0.000 & 0.000 & 0.000 & 0.000 & 0.000 & 0.000 & 0.000 & 0.000 & 0.000 \\
\hline $\mathrm{Cr}$ & 0.000 & 0.000 & 0.000 & 0.000 & 0.000 & 0.000 & 0.000 & 0.000 & 0.000 & 0.000 \\
\hline $\mathrm{Fe}^{3+}$ & 0.679 & 0.000 & 0.000 & 0.118 & 0.000 & 0.000 & 1.348 & 0.000 & 1.596 & 1.007 \\
\hline $\mathrm{Fe}^{2+}$ & 2.714 & 2.591 & 2.370 & 2.512 & 2.698 & 2.474 & 1.329 & 2.707 & 1.311 & 1.922 \\
\hline $\mathrm{Mn}$ & 0.000 & 0.000 & 0.000 & 0.000 & 0.000 & 0.000 & 0.000 & 0.000 & 0.000 & 0.000 \\
\hline $\mathrm{Mg}$ & 2.161 & 2.488 & 2.047 & 1.844 & 2.123 & 2.236 & 1.793 & 2.358 & 2.002 & 2.108 \\
\hline $\mathrm{Ca}$ & 0.899 & 0.634 & 0.857 & 1.227 & 1.173 & 1.153 & 0.710 & 1.267 & 0.608 & 0.818 \\
\hline $\mathrm{Na}$ & 0.000 & 0.000 & 0.000 & 0.000 & 0.000 & 0.000 & 0.000 & 0.000 & 0.000 & 0.000 \\
\hline $\mathrm{K}$ & 0.000 & 0.000 & 0.000 & 0.000 & 0.000 & 0.000 & 0.000 & 0.000 & 0.000 & 0.000 \\
\hline Sum & 10.057 & 9.107 & 8.888 & 9.072 & 9.248 & 9.182 & 8.503 & 9.416 & 8.610 & 8.927 \\
\hline
\end{tabular}


Table 4. Cont.

\begin{tabular}{|c|c|c|c|c|c|c|c|c|c|c|}
\hline \multirow{3}{*}{\begin{tabular}{|l|} 
Sample \\
Mineral \\
Positions
\end{tabular}} & \multicolumn{10}{|c|}{$17615-11$} \\
\hline & \multicolumn{10}{|c|}{ Actinolite } \\
\hline & 1 & 2 & 3 & 4 & 5 & 6 & 7 & 8 & 9 & 10 \\
\hline $\mathrm{Na}_{2} \mathrm{O}$ & 0.00 & 0.00 & 0.00 & 0.00 & 0.00 & 0.00 & 0.00 & 0.00 & 0.00 & 0.00 \\
\hline $\mathrm{MgO}$ & 11.77 & 10.97 & 13.17 & 12.08 & 13.06 & 13.92 & 12.77 & 13.09 & 13.28 & 13.15 \\
\hline $\mathrm{Al}_{2} \mathrm{O}_{3}$ & 5.39 & 3.54 & 9.69 & 9.18 & 7.61 & 9.68 & 8.86 & 4.45 & 3.17 & 2.43 \\
\hline $\mathrm{SiO}_{2}$ & 52.89 & 46.16 & 44.10 & 47.52 & 43.23 & 42.82 & 45.86 & 42.54 & 44.05 & 51.73 \\
\hline $\mathrm{K}_{2} \mathrm{O}$ & 0.00 & 0.00 & 0.00 & 0.00 & 0.00 & 0.00 & 0.00 & 0.00 & 0.00 & 0.00 \\
\hline $\mathrm{CaO}$ & 10.87 & 11.73 & 13.83 & 12.17 & 10.52 & 9.96 & 10.59 & 11.65 & 12.13 & 11.48 \\
\hline $\mathrm{TiO}_{2}$ & 0.00 & 0.00 & 0.00 & 0.00 & 0.00 & 0.00 & 0.00 & 0.00 & 0.00 & 0.00 \\
\hline $\mathrm{MnO}$ & 0.00 & 0.00 & 0.00 & 0.00 & 0.00 & 0.00 & 0.00 & 0.00 & 0.00 & 0.00 \\
\hline $\mathrm{FeO}_{\mathrm{T}}$ & 18.15 & 12.82 & 14.48 & 13.63 & 12.04 & 14.98 & 19.77 & 14.76 & 12.02 & 11.01 \\
\hline $\mathrm{Cr}_{2} \mathrm{O}_{3}$ & 0.00 & 0.00 & 0.00 & 0.00 & 0.00 & 0.00 & 0.00 & 0.00 & 0.00 & 0.00 \\
\hline Total & 99.07 & 85.22 & 95.27 & 94.58 & 86.46 & 91.36 & 97.85 & 86.49 & 84.65 & 89.80 \\
\hline $\mathrm{Si}$ & 7.62 & 7.39 & 6.42 & 7.07 & 6.97 & 6.54 & 6.67 & 6.82 & 7.16 & 7.99 \\
\hline $\mathrm{Ti}$ & 0.00 & 0.00 & 0.00 & 0.00 & 0.00 & 0.00 & 0.00 & 0.00 & 0.00 & 0.00 \\
\hline $\mathrm{Al}$ & 0.915 & 0.669 & 1.664 & 1.611 & 1.447 & 1.744 & 1.518 & 0.841 & 0.607 & 0.443 \\
\hline $\mathrm{Cr}$ & 0.000 & 0.000 & 0.000 & 0.000 & 0.000 & 0.000 & 0.000 & 0.000 & 0.000 & 0.000 \\
\hline $\mathrm{Fe}^{3+}$ & 0.000 & 1.717 & 1.763 & 0.244 & 0.609 & 1.172 & 1.153 & 1.979 & 1.633 & 0.000 \\
\hline $\mathrm{Fe}^{2+}$ & 2.186 & 0.000 & 0.000 & 1.452 & 1.015 & 0.742 & 1.250 & 0.000 & 0.000 & 1.423 \\
\hline $\mathrm{Mn}$ & 0.000 & 0.000 & 0.000 & 0.000 & 0.000 & 0.000 & 0.000 & 0.000 & 0.000 & 0.000 \\
\hline $\mathrm{Mg}$ & 2.527 & 2.619 & 2.858 & 2.679 & 3.139 & 3.170 & 2.766 & 3.128 & 3.215 & 3.028 \\
\hline $\mathrm{Ca}$ & 1.678 & 2.013 & 2.158 & 1.941 & 1.818 & 1.631 & 1.649 & 2.001 & 2.112 & 1.901 \\
\hline $\mathrm{Na}$ & 0.000 & 0.000 & 0.000 & 0.000 & 0.000 & 0.000 & 0.000 & 0.000 & 0.000 & 0.000 \\
\hline $\mathrm{K}$ & 0.000 & 0.000 & 0.000 & 0.000 & 0.000 & 0.000 & 0.000 & 0.000 & 0.000 & 0.000 \\
\hline Sum & 14.925 & 14.972 & 15.458 & 15.081 & 15.202 & 15.393 & 15.386 & 15.434 & 15.266 & 14.787 \\
\hline
\end{tabular}

Note: element compositions were determined using energy dispersive spectrometer (EDS) method at institute of Geology, Chinese Academy of Geological Sciences.

Mineral within sample 17615-3 displays $\mathrm{MgO}$ contents ranges from 0.03 to $0.93 \mathrm{wt} \%$ (average value $0.50 \%$ ). $\quad \mathrm{Al}_{2} \mathrm{O}_{3}$ contents ranges from 18.89 to $23.29 \mathrm{wt} \%$ (average value $21.11 \%$ ). $\mathrm{SiO}_{2}$ contents ranges from 31.09 to $35.51 \mathrm{wt} \%$ (average value $33.64 \%$ ). $\mathrm{CaO}$ and $\mathrm{FeO}_{\mathrm{T}}$ contents range from 23.09 to $27.54 \mathrm{wt} \%$ (average value $24.72 \%$ ) and from 8.37 to $11.81 \mathrm{wt} \%$ (average value $9.63 \%$ ), respectively. The chemical compositions are close to common epidote with an approximate mineral formula that can be inferred as $\mathrm{Ca}_{3} \mathrm{FeAl}_{2}\left[\mathrm{SiO}_{4}\right]\left[\mathrm{Si}_{2} \mathrm{O}_{7}\right] \mathrm{O}(\mathrm{OH})_{X}[23]$.

Mineral within sample 17615-4 displays $\mathrm{MgO}$ contents ranges from 10.57 to 16.97 wt \% (average value $13.47 \%$ ). $\quad \mathrm{Al}_{2} \mathrm{O}_{3}$ contents ranges from 3.21 to $9.82 \mathrm{wt} \%$ (average value $6.23 \%$ ). $\mathrm{SiO}_{2}$ contents ranges from 45.53 to $55.85 \mathrm{wt} \%$ (average value $49.92 \%$ ). $\mathrm{CaO}$ and $\mathrm{FeO}_{\mathrm{T}}$ contents range from 9.29 to $11.84 \mathrm{wt} \%$ (average value $10.44 \%$ ) and from 12.28 to $18.65 \mathrm{wt} \%$ (average value $15.07 \%$ ), respectively. The chemical compositions are close to common actinolite with an approximate mineral formula that can be inferred as $\mathrm{Ca}_{2}(\mathrm{Mg}, \mathrm{Fe})_{5}\left(\mathrm{Si}_{4} \mathrm{O}_{11}\right)_{2}(\mathrm{OH})_{X}[23]$.

Mineral within sample 17615-6 displays $\mathrm{Na}_{2} \mathrm{O}$ contents ranges from 1.14 to $7.23 \mathrm{wt} \%$ (average value $4.16 \%$ ). $\mathrm{MgO}$ contents ranges from 8.16 to $14.54 \mathrm{wt} \%$ (average value $10.91 \%$ ). $\mathrm{Al}_{2} \mathrm{O}_{3}$ contents ranges from 9.52 to 13.71 wt \% (average value $11.22 \%$ ). $\mathrm{SiO}_{2}$ and $\mathrm{CaO}$ contents range from 48.25 to $54.85 \mathrm{wt} \%$ (average value $51.15 \%$ ) and from 1.41 to $9.72 \mathrm{wt} \%$ (average value $5.78 \%$ ), respectively. $\mathrm{TiO}_{2}$ and $\mathrm{FeO}_{\mathrm{T}}$ contents range from 0.05 to 2.96 wt \% (average value $0.79 \%$ ) and from 10.35 to $19.46 \mathrm{wt} \%$ (average value $14.07 \%$ ), respectively. The chemical compositions are close to common glaucophane with an approximate mineral formula that can be inferred as $\mathrm{Na}_{2}(\mathrm{Mg}, \mathrm{Fe})_{3} \mathrm{Al}_{2} \mathrm{Si}_{8} \mathrm{O}_{22}(\mathrm{OH})_{X}[23]$. 
Mineral within sample 17615-7-2 shows: $\mathrm{Al}_{2} \mathrm{O}_{3}$ contents ranges from 31.19 to 37.96 wt \% (average value $35.06 \%$ ). $\mathrm{SiO}_{2}$ contents ranges from 39.49 to $40.91 \mathrm{wt} \%$ (average value $40.24 \%$ ). $\mathrm{CaO}$ and $\mathrm{FeO}_{\mathrm{T}}$ contents range from 20.81 to $25.73 \mathrm{wt} \%$ (average value $22.09 \%$ ) and from 0.02 to $0.65 \mathrm{wt} \%$ (average value $0.27 \%$ ). The chemical compositions are close to common zoisite with an approximate mineral formula that can be inferred as $\mathrm{Ca}_{2} \mathrm{Al}_{3}\left[\mathrm{SiO}_{4}\right]\left[\mathrm{Si}_{2} \mathrm{O}_{7}\right] \mathrm{O}(\mathrm{OH})_{\mathrm{X}}[23]$.

Mineral within sample 17615-10 displays $\mathrm{MgO}$ contents ranges from 9.21 to $12.38 \mathrm{wt} \%$ (average value $10.91 \%$ ). $\mathrm{Al}_{2} \mathrm{O}_{3}$ contents ranges from 20.95 to $29.66 \mathrm{wt} \%$ (average value $25.01 \%$ ). $\mathrm{SiO}_{2}$ contents ranges from 22.89 to $29.53 \mathrm{wt} \%$ (average value $25.57 \%$ ). $\mathrm{CaO}$ and $\mathrm{FeO}_{\mathrm{T}}$ contents range from 4.39 to $9.69 \mathrm{wt} \%$ (average value $6.74 \%$ ) and from 20.92 to $27.45 \mathrm{wt} \%$ (average value $25.16 \%$ ), respectively. The chemical compositions are close to common chlorite and have an approximate mineral formula that can be inferred as $(\mathrm{Mg}, \mathrm{Fe}, \mathrm{Al})_{6}\left[(\mathrm{Si}, \mathrm{Al}) \mathrm{O}_{10}\right](\mathrm{OH})_{X}[23]$.

Mineral within sample 17615-2 shows similar chemical compositions with 17615-4: MgO contents ranges from 10.97 to $13.92 \mathrm{wt} \%$ (average value $12.73 \%$ ). $\mathrm{Al}_{2} \mathrm{O}_{3}$ contents ranges from 2.43 to $9.69 \mathrm{wt} \%$ (average value $6.40 \%$ ). $\mathrm{SiO}_{2}$ contents ranges from 42.54 to $52.89 \mathrm{wt} \%$ (average value $46.09 \%$ ). $\mathrm{CaO}$ and $\mathrm{FeO}_{\mathrm{T}}$ contents range from 9.96 to $13.83 \mathrm{wt} \%$ (average value $11.49 \%$ ) and from 11.01 to $19.77 \mathrm{wt} \%$ (average value $14.37 \%$ ), respectively. It is also close to the chemical compositions of common actinolite with an approximate mineral formula that can be inferred as $\mathrm{Ca}_{2}(\mathrm{Mg}, \mathrm{Fe})_{5}\left(\mathrm{Si}_{4} \mathrm{O}_{11}\right)_{2}(\mathrm{OH})_{\mathrm{X}}[23]$.

\subsection{Reflectance Characteristics}

The results of the white reference tests, which can detect any meaningful variation in the standard spectra, are shown in Figure 6. There was no significant change during the experiment. Figure 7 presents the spectra of eight rock samples from the Kaishantun ophiolite suite. Six spectrum measurements from different parts of each sample showed no variation of absorption features during the measurements. Mineral types had distinctive absorption features and similar shapes, and the spectra of different parts of each rock sample were not identical (Figure 7).

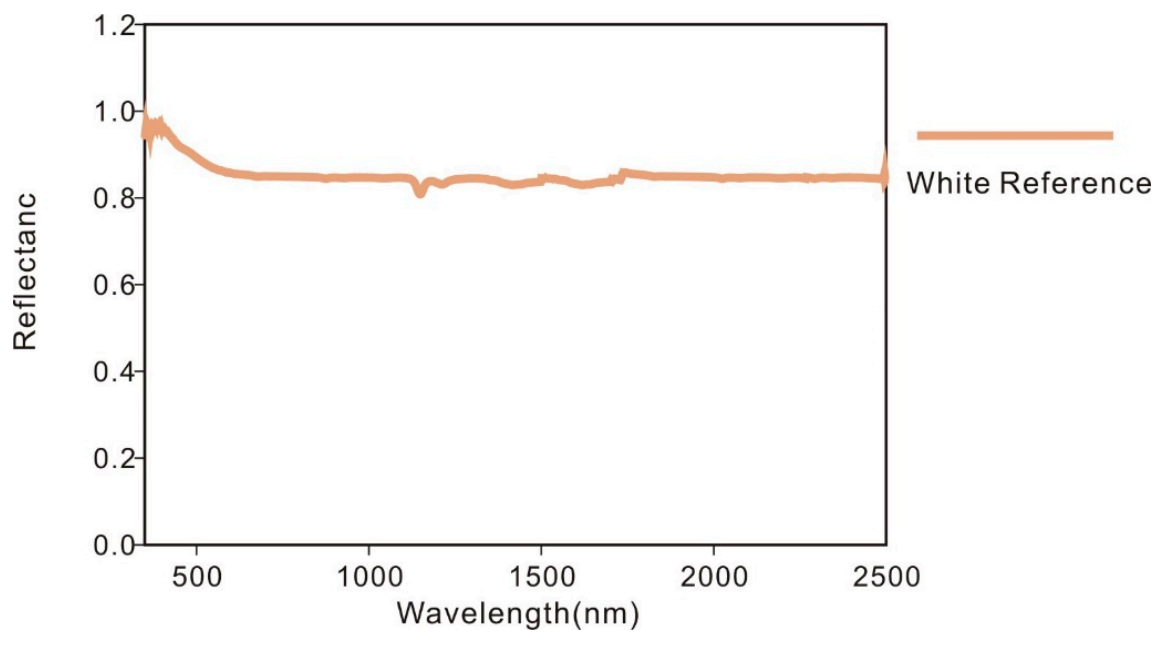

Figure 6. Spectral of white reference before and after the experiment. 

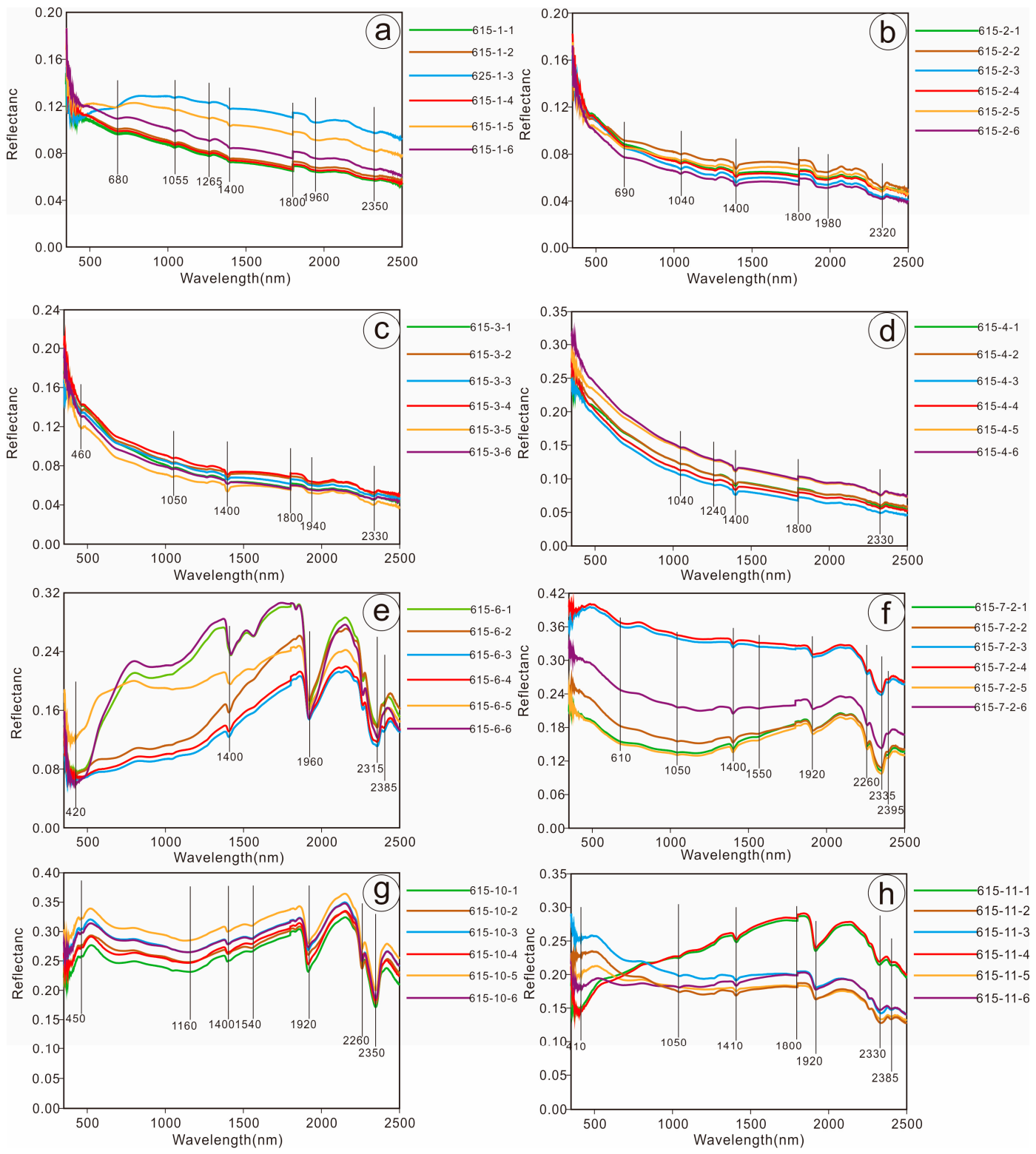

Figure 7. Spectra of eight ophiolite samples recorded by analytical spectral devices (ASD) spectrometer. (a) 17615-1; (b) 17615-2; (c) 17615-3; (d) 17615-4; (e) 17615-6; (f) 17615-7-2; (g) 17615-10; and (h) 17615-11. Lines $1-6$ are the six data points tested for the vote.

One peridotite sample (17615-1) showed absorption peaks at 675, 1045, 1265, 1400, and $2330 \mathrm{~nm}$. Three other peridotite samples (17615-2, 17615-4, and 17615-6) had similar absorption peaks, but $17615-3$ had a $480-n m$ peak. One diabase sample (17615-6) displayed absorption peaks at 420, 1400, 1940, and $2330 \mathrm{~nm}$. One basalt sample (17615-7-2) had absorption peaks at 710, 1400, 1940, 2260, and $2350 \mathrm{~nm}$. The pyroxenite sample (17615-10) also showed absorption at 450, 1160, 1400, 1540, 1930, 2260, and $2350 \mathrm{~nm}$. One gabbro sample (17615-11) displayed absorption at 420, 1050, 1400, 1800, 1930, and $2345 \mathrm{~nm}$. Table 5 includes all spectral absorption features from these tests. 
Table 5. Summary of Mineral Absorption Peaks from This Study, with Reference Samples from the United States Geological Survey Spectral Librar.

\begin{tabular}{ccc}
\hline Samples & Absorption(s) @ nm & Reference \\
\hline $17615-1$ (Peridotite) & $680,1055,1265,1400,1960,2350$ & this study \\
$17615-2$ (Peridotite) & $690,1040,1400,1800,1980,2320$ & this study \\
$17615-3$ (Peridotite) & $450,1400,1800,1940,2330$ & this study \\
$17615-4$ (Peridotite) & $1040,1240,1400,1800,2350$ & this study \\
$17615-6$ (Diabase) & $420,1400,1960,2315,2385$ & this study \\
$17615-7-2$ (Basalt) & $610,1050,1400,1940,1550,1920,2360,2335,2395$ & this study \\
$17615-10$ (Pyroxenite) & $450,1160,1400,1540,1920,2260,2350$ & this study \\
$17615-11$ (Gabbro) & $410,1050,1410,1800,1920,2330,2385$ & this study \\
Serpentine & $470,1410,2350$ & USGS [25] \\
Zoisite & $720,1550,1800,2310,2375$ & USGS [25] \\
Chlorite & $660,1410,1950,2350$ & USGS [25] \\
Epidote & $480,610,1045,1545,2200,2235$ & USGS [25] \\
Actinolite & 1950,2350 & USGS [25] \\
Glaucophane & $1405,1945,2315,2386$ & USGS [25] \\
\hline
\end{tabular}

\section{Discussion}

As the dominant species used for type differentiation of ophiolite suites, it is important to be able to distinguish typical minerals such as actinolite, epidote, zoisite, and glaucophane from the overall mineral assemblage in rocks. However, it is difficult to distinguish them from associated minerals and also from other femic-ultramafic minerals in the ophiolite suite, as they possess identical tectonic dynamic setting and various degrees of alteration. The traditional techniques, such as microscopic identification and the dissolution method with manual sorting, are inefficient, uneconomical, and time-consuming. Fortunately, multispectral remote sensing based on mineral spectra is a promising tool for identification of different lithologies and altered minerals.

Under normal solar spectrum $(300-2500 \mu \mathrm{m})$, several minerals have distinct absorption features because of electronic transition, vibrational overtones, charge transfer and conduction. Actinolite has a distinct $\mathrm{Fe}, \mathrm{Mg}-\mathrm{OH}$ combination absorption feature within the 2300-2350 nm range and a less intense absorption feature at $1400 \mathrm{~nm}$ depending on electronic transition processes $\left(\mathrm{Fe}^{2+}\right)$ (Figure $8 \mathrm{a}$ ). Epidote shows distinct $2200 \mathrm{~nm}$ absorption and a less intense $2235 \mathrm{~nm}$ absorption feature due to the $\mathrm{Al}-\mathrm{OH}$ bond and Fe-Mg content (Figure 8a). Similarly, glaucophane exhibits intense absorption feature at $1945 \mathrm{~nm}$ and $1400 \mathrm{~nm}$ and a weak absorption feature within the 2315-2386 nm range, derived from its Fe-Mg content (Figure 8a). Chlorite displays pronounced absorption at 2310-2330 nm caused by the $\mathrm{Fe}-\mathrm{Mg}$ content and a shallow absorption feature at $1950 \mathrm{~nm}$ (Figure $8 \mathrm{~b}$ ). Zoisite has a strong absorption at $720 \mathrm{~nm}$ and a less intense absorption feature at $1800 \mathrm{~nm}$ and within the $2310-2375 \mathrm{~nm}$ range due to $\mathrm{Fe}$, $\mathrm{Mg}-\mathrm{OH}$ and $\mathrm{Al}-\mathrm{OH}$ bond (Figure $8 \mathrm{~b}$ ). Moreover, serpentine shows a strong $\mathrm{Fe}, \mathrm{Mg}-\mathrm{OH}$ absorption feature at $2350 \mathrm{~nm}$, a less intense absorption feature at $1410 \mathrm{~nm}$, and a weak absorption feature at $470 \mathrm{~nm}$ depending on electronic transition processes $\left(\mathrm{Fe}^{3+}\right)$ (Figure $8 \mathrm{~b}$ ). 

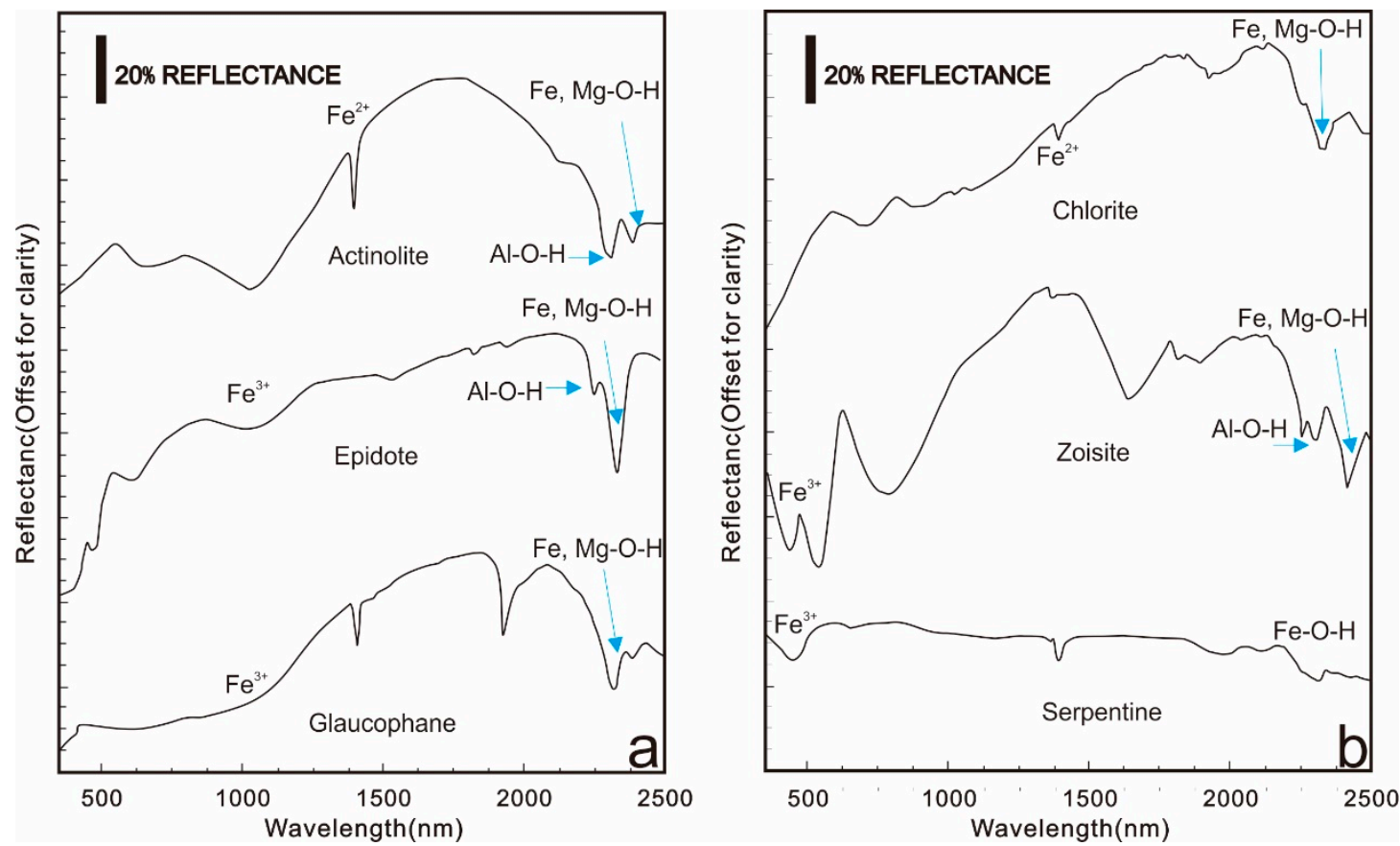

Figure 8. Laboratory spectra of: (a) actinolite, epidote and chlorite and glaucophane; and (b) chlorite, zoisite and serpentine (USGS spectral library).

The reflectance of peridotite samples (sample 17615-1, -2, -3, and -4) from Kaishantun ophiolite suite showed strong absorption features at 1400, and 2350. Their shapes compared with standard single mineral spectra presented in the USGS spectral library [25]. The spectral curves were similar to the spectra of serpentines (intense absorption feature) and chlorites and minor signatures of epidotes. Sample 17615-1 had higher reflection values, emphasizing that it has more leucocratic minerals, such as plagioclase, but its standard absorption peaks and shapes suggest that there is no difference in its primary mineral components. To confirm the availability of these absorption features, chemical compositions of the minerals in the ophiolite samples were presented. Samples 17615-1 and 17615-2 had minerals with serpentine features, while 17615-3 contains epidote and sample 17615-4 has minerals closer to actinolite. The results were consistent with the microscopic observation.

In addition, according to the spectral absorption features and shapes reported in this study, sample 17615-6 shows a distinct absorption feature at $1960 \mathrm{~nm}$, a less intense absorption feature within the 2135-2385 nm range, and a minor absorption feature at $1400 \mathrm{~nm}$ and $420 \mathrm{~nm}$, which are attributed to the existence of glaucophanes, actinolites and chlorites. Sample 17615-7-2 exhibits pronounced absorption at $1920 \mathrm{~nm}$ and $2350 \mathrm{~nm}$ and spectral curves were similar to the spectra of epidotes and minor actinolites. Sample 17615-10 showed an intense absorption features at $1920 \mathrm{~nm}$, a less strong absorption feature within the 2260-2350 $\mathrm{nm}$ range and a minor absorption feature at $1400 \mathrm{~nm}, 1160 \mathrm{~nm}$ and $450 \mathrm{~nm}$, which are due to the presence of glaucophanes, zoisites and actinolites. Sample 17615-11 has a distinct absorption feature within the $2330-2385 \mathrm{~nm}$ range, a less strong absorption feature at $1920 \mathrm{~nm}$, and a weak absorption feature at $1410 \mathrm{~nm}, 1050 \mathrm{~nm}$ and $410 \mathrm{~nm}$, the spectral curves were similar to the spectra of actinolites (intense absorption feature) and minor signatures of zoisites. These absorption features are in accordance with chemical compositions of the minerals. In chemical composition, samples 17615-6 and 17615-7-2 had minerals with glaucophane and zoisite features, respectively. Samples 17615-10 and 17615-4 has chlorite and actinolite minerals. The results are correspond to microscopic observation.

Therefore, we can conclude that both the blueschist facies (glaucophane + epidote + chlorite) and the greenschist facies (actinolite + epidote + chlorite) exist in the Kaishantun ophiolite suite. $\mathrm{Wu}$ (2003) reported that chloritoid + carpholite + phengite and actinolite + zoisite + barroisite mineral assemblages 
were found as a blueschist facie in the northern part of Kaishantun ophiolite suite, but they did not discuss the implications of the presence of these minerals [26]. Blueschist forms in high-pressure, low-temperature environments, and regions associated with subduction of oceanic material beneath either oceanic or continental crust are characterized by blueschist and greenschist facies [27]. Although some blueschists could also be produced in the collision zone, discrimination about the formation of their environment needs to consider the regional tectonic setting [27]. Kaishantun ophiolite suite is located at eastern part of Xar Moron-Changchun-Yanji Fault due to the subduction of the Paleo-Asian Ocean in the Late Permian [13,28,29]. Tang (2007) reported the SHRIMP age 286 Ma of granite pebbles in Kaishantun ophiolite suite [30]. They are, therefore, typical signs of subduction tectonics [26]. It is deduced that Kaishantun ophiolite suite mainly experienced the blueschist facies metamorphic peak during subduction and greenschist facies retrograde metamorphism during later slab rollback.

\section{Conclusions}

1 We measured the spectra of main type samples from the Kaishantun ophiolite suite in Northeast China. Peridotite showed absorption peaks at 480, 675, 1045, 1265, 1400, and $2330 \mathrm{~nm}$. Diabase had absorption peaks at 420,1400, 1940, and $2330 \mathrm{~nm}$. Basalt had absorption peaks at 710, 1400, 1940, 2260, and $2350 \mathrm{~nm}$. Pyroxenite showed absorption at 450, 1160, 1400, 1540, 1930, 2260, and $2350 \mathrm{~nm}$. Gabbro displayed absorption at 420, 1050, 1400, 1800, 1930, and $2345 \mathrm{~nm}$.

2 Glaucophane $(1405,1945,2315$, and $2386 \mathrm{~nm})$, actinolite (1950 and $2350 \mathrm{~nm})$, zoisite $(720,1550$, 1800, 2310 and $2375 \mathrm{~nm}$ ) and epidote $(480,600,1050,1550$, and $1950 \mathrm{~nm})$ were used to distinguish these samples from other associated minerals in ophiolite samples.

3 In conjunction with its chemical composition, the blueschist facies (glaucophane + epidote + chlorite) and the greenschist facies (actinolite + epidote + chlorite) mineral assemblage can be recognized based on their spectral signatures.

4 Considered about the regional tectonic setting, the Kaishantun ophiolite suite probably experienced the blueschist facies metamorphic peak during subduction and greenschist facies retrograde metamorphism during later slab rollback.

Supplementary Materials: The following are available online at http://www.mdpi.com/2075-163X/8/3/100/s1.

Acknowledgments: This work was financially supported by Geological Tectonic Division and Comprehensive Integration of Regional Geological Survey of China (Grant No. DD20160345). We gratefully extend our appreciation to Lijun Nie, who gave us much help and advice during the fieldwork and Hongliang Zhang, who helped us deal with spectral data. The authors would like to thank Enago for the English language review and the anonymous reviewers for their constructive criticism and useful comments.

Author Contributions: Xiaozhong Ding and Yanxue Liu formulated the problem, organized the research team, and guided the study and interpretation of results. Chenglong Shi contributed to interpretations of the samples and SEM-EDX analyses. Xiaodong Zhou participated and contributed to the fieldwork. All authors participated in writing the manuscript.

Conflicts of Interest: The authors declare no conflict of interest.

\section{References}

1. Santosh, M.; Teng, X.M.; He, X.F.; Tang, L.; Yang, Q.Y. Discovery of Neoarchean suprasubduction zone ophiolite suite from Yishui complex in the north China craton. Gondwana Res. 2015, 38, 1-27. [CrossRef]

2. Cawood, P.A.; Kroner, A.; Collins, W.J.; Kusky, T.M.; Mooney, W.D.; Windley, B.F. Accretionary orogens through Earth history. Geol. Soc. Speci. 2009, 318, 1-36. [CrossRef]

3. Dilek, Y.; Flower, M.F.J. Arc-trench rollback and forearc accretion: 2. A model template for ophiolites in albania, cyprus, and oman. Geol. Soc. Lond. 2003, 218, 43-68. [CrossRef]

4. Babu, P.S.; Majumdar, T.J.; Bhattacharya, A.K. Study of spectral signatures for exploration of bauxite ore deposits in Panchpatmali, India. Geocarto Int. 2015, 30, 545-559. [CrossRef] 
5. Boesche, N.; Rogass, C.; Lubitz, C.; Brell, M.; Herrmann, S.; Mielke, C.; Tonn, S.; Appelt, O.; Altenberger, U.; Kaufmann, H. Hyperspectral REE (rare earth element) mapping of outcrops-Applications for Neodymium detection. Remote Sens. 2015, 7, 5160-5186. [CrossRef]

6. Harris, J.R.; Mcgregor, R.; Budkewitsch, P. Geological analysis of hyperspectral data over southwest Baffin Island: Methods for producing spectral maps that relate to variations in surface lithologies. Can. J. Remote Sens. 2010, 36, 412-435. [CrossRef]

7. Nair, A.M.; Mathew, G. Effect of bulk chemistry in the spectral variability of igneous rocks in VIS-NIR region: Implications to remote compositional mapping. Int. J. Appl. Earth Obs. Geoinform. 2014, 30, 227-237. [CrossRef]

8. Jilin Bureau of Geology and Mineral Resources (JBGMR). Regional Geology of Jilin Province; Geological Publishing House: Beijing, China, 1988; pp. 1-698, (In Chinese with English summary).

9. Jilin Bureau of Geology and Mineral Resources (JBGMR). Stratigraphy (Lithostatic) of Jilin Province; China University of Geosciences Press: Wuhan, China, 1997; pp. 1-324, (In Chinese with English summary).

10. Wu, F.Y.; Jahn, B.M.; Wilde, S.A.; Sun, D.Y. Phanerozoic continental crustal growth: Sr-Nd isotopic evidence from the granites in Northeastern China. Tectonophysics 2000, 328, 87-113. [CrossRef]

11. Wu, F.Y.; Sun, D.Y.; Li, H.; Jahn, B.M.; Wilde, S. A-type granites in Northeastern China: Age and geochemical constraints on their petrogenesis. Chem. Geol. 2002, 187, 143-173. [CrossRef]

12. Grebennikov, A.V. A-type granites and related rocks: Petrogenesis and classification. Rus. Geol. Geophys. 2014, 55, 1074-1086. [CrossRef]

13. Li, J.Y. Permian geodynamic setting of Northeast China and adjacent regions: Closure of the Paleo-Asian Ocean and subduction of the Paleo-Pacific Plate. J. Asian Earth. Sci. 2006, 26, 207-224. [CrossRef]

14. Han, B.F.; Kagami, H.; Li, H.M. Age and Nd-Sr isotopic geochemistry of the Guangtoushan alkaline granite, Hebei Province, China; Implications for Early Mesozoic crust-mantle interaction in North China Block. Acta Petrol. Sin. 2004, 20, 1376-1388.

15. Ionov, D.A.; Hofmann, A.W. Nb Ta-rich mantle amphiboles and micas: Implications for subduction-related metasomatic trace element fractionations. Earth Planet. Sci. Lett. 1995, 131, 341-356. [CrossRef]

16. Wu, F.Y.; Zhao, G.C.; Sun, D.Y.; Wilde, S.A.; Yang, J.H. The Hulan group: Its role in the evolution of the Central Asian Orogenic Belt of NE China. J. Asian Earth. Sci. 2007, 30, 542-556. [CrossRef]

17. Wilde, S.A. Final amalgamation of the Central Asian Orogenic Belt in ne china: Paleo-Asian Ocean closure versus Paleo-Pacific Plate subduction-A review of the evidence. Tectonophysics 2015, 662, 345-362. [CrossRef]

18. Xiao, W.J.; Windley, B.F.; Sun, S.; Li, J.L.; Huang, B.C.; Han, C.M.; Yuan, C.; Sun, M.; Chen, H.L. A tale of amalgamation of three Permo-Triassic collage systems in central Asia: Oroclines, sutures, and terminal accretion. Annu. Rev. Earth Planet. Sci. 2015, 43, 477-507. [CrossRef]

19. Yang, D.-G.; Sun, D.-Y.; Gou, J.; Hou, X.-G. U-Pb ages of zircons from Mesozoic intrusive rocks in the Yanbian area, Jilin Province, NE China: Transition of the Paleo-Asian oceanic regime to the Circum-Pacific tectonic regime. J. Asian Earth Sci. 2017, 143, 171-190. [CrossRef]

20. Zhou, J.B.; Han, J.; Zhao, G.C.; Zhang, X.Z.; Cao, J.L.; Wang, B.; Pei, S.H. The emplacement time of the Hegenshan ophiolite: Constraints from the unconformably overlying Paleozoic strata. Tectonophysics 2015, 662, 398-415. [CrossRef]

21. Shao, J.A.; Tang, K.D. The ophiolite melange in Kaishantun, Jilin Province, China. Acta Petrol. Sin. 1995, 11, 219, (In Chinese with English summary).

22. Shao, J.A.; Tang, K.D. Reconstruction of an ancient continental margin and its tectonic significance: New progress in Yanbian geological research. Sci. China Ser. B 1995, 25, 548-555. (In Chinese)

23. Deer, W.; Howie, R.A.; Zussman, A.J. An Introduction to the Rock-Forming Minerals; Longman Scientific \& Technical: London, UK, 1996.

24. Holland, T.; Powell, R. Activity-composition relations for phases in petrological calculations: An asymmetric multicomponent formulation. Contrib. Mineral. Petr. 2003, 145, 492-501. [CrossRef]

25. Clark, R.N.; Swayze, G.A.; Gallagher, A.J.; King, T.V.V.; Calvin, W.M. The U.S. Geological Survey, Digital Spectral Library: Version 1: 0.2 to 3.0 Microns; U.S. Geological Survey Open File Report; U.S. Geological Survey: Reston, VA, USA, 1993. 
26. Wu, H.Q.; Tang, K.D.; Li, C.Y. The assemblage chloritoid+carpholite+phengite in low-grade metamorphic rocks in Kaishantun area, Yanbian, Jilin Province-The evidence of blueschist facies metamorphis. Geol. B. China 2003, 9, 651-654, (In Chinese with English summary).

27. Stern, R.J. Evidence from ophiolites, blueschists, and ultrahigh-pressure metamorphic terranes that the modern episode of subduction tectonics began in Neoproterozoic time. Geology 2005, 33, 557-560. [CrossRef]

28. Deyou, S.; Fuyuan, W.; Shan, G.; Xiaopin, L. Confirmation of two episodes of A-type granite emplacement during Late Triassic and Early Jurassic in the Central Jilin Province, and their constraints on the structural pattern of eastern Jilin-Heilongjiang area, China. Earth Sci. Front. 2005, 2, 263-275, (In Chinese with English summary).

29. Li, C.D.; Zhang, F.Q.; Miao, L.C.; Xie, H.Q.; Xu, Y.W. Zircon SHRIMP geochronology and geochemistry of late permian high-Mg Andesites in seluohe area, Jilin Province, China. Acta Petrol. Sin. 2007, 23, 767-776, (In Chinese with English summary).

30. Tang, K.D.; Zhao, A.L. New evidence of Palaeozoic stratigraphy in the Kaishantun area, Yanbian, Jilin. J. Stratigr. 2007, 31, 142-150, (In Chinese with English summary).

(C) 2018 by the authors. Licensee MDPI, Basel, Switzerland. This article is an open access article distributed under the terms and conditions of the Creative Commons Attribution (CC BY) license (http://creativecommons.org/licenses/by/4.0/). 